\title{
Bayesian Nonparametric Learning of How Skill Is Distributed across the Mutual Fund Industry
}

\author{
Mark Fisher, Mark J. Jensen, and Paula Tkac
}

\section{Working Paper 2019-3 \\ March 2019}

\begin{abstract}
In this paper, we use Bayesian nonparametric learning to estimate the skill of actively managed mutual funds and also to estimate the population distribution for this skill. A nonparametric hierarchical prior, where the hyperprior distribution is unknown and modeled with a Dirichlet process prior, is used for the skill parameter, with its posterior predictive distribution being an estimate of the population distribution. Our nonparametric approach is equivalent to an infinitely ordered mixture of normals where we resolve the uncertainty in the mixture order by partitioning the funds into groups according to the group's average ability and variability. Applying our Bayesian nonparametric learning approach to a panel of actively managed, domestic equity funds, we find the population distribution of skill to be fat-tailed, skewed towards higher levels of performance. We also find that it has three distinct modes: a primary mode where the average ability covers the average fees charged by funds, a secondary mode at a performance level where a fund loses money for its investors, and lastly, a minor mode at an exceptionally high skill level.
\end{abstract}

JEL classification: G11 ,C11, C14

Key words: Bayesian nonparametrics, mutual funds, unsupervised learning

https://doi.org/10.29338/wp2019-03

The authors thank Vikas Agarwal, Wayne Ferson, Kris Gerardi, Siva Nathan, Jay Shanken. and the conference participants at the 10th Annual All Georgia Finance Conference, the 2014 Southern Finance Meetings, the Economics, Finance, and Business Section of ISBA Bayes 250, the 2014 Bayesian Workshop of the Remini Centre of Economic Analysis, the 2015 NBERNSF Seminar on Bayesian Inference in Econometrics and Statistics, the 2015 European Seminar on Bayesian Econometrics, the 2017 ISBA Conference on Bayesian Nonparametric, the 2018 Workshop on Bayesian Methods in Finance held at the ESSEC Business School, and the department members at the Institution of Statistics and Mathematics at Vienna University of Business and Economics, the DeGroote School of Business at McMaster University, Clemson University, the University of North Carolina-Charlotte, Queen Mary-University of London, and the University of Montreal for their helpful comments and suggestions. The views expressed here are those of the authors and not necessarily those of the Federal Reserve Bank of Atlanta or the Federal Reserve System. Any remaining errors are the authors' responsibility.

Please address questions regarding content to Mark Fisher, Research Department, Federal Reserve Bank of Atlanta, 1000 Peachtree Street NE, Atlanta, GA 30309-4470, 404-498-8757, mark@markfisher.net; Mark J. Jensen, Research Department, Federal Reserve Bank of Atlanta, 1000 Peachtree Street NE, Atlanta, GA 30309-4470, 404-498-8019, mark.jensen@atl.frb.org; or Paula Tkac, Research Department, Federal Reserve Bank of Atlanta, 1000 Peachtree Street NE, Atlanta, GA 30309-4470, 404-498-8813, paula.tkac@atl.frb.org.

Federal Reserve Bank of Atlanta working papers, including revised versions, are available on the Atlanta Fed's website at www.frbatlanta.org. Click "Publications" and then "Working Papers." To receive e-mail notifications about new papers, use frbatlanta.org/forms/subscribe. 


\section{Introduction}

Since the seminal article by Jensen (1968) estimating the skill level of managed mutual funds has been widely researched and debated (see Elton \& Gruber 2013). In addition to measuring the skill of a fund, others have investigated how skill is the distributed across the industry. For instance, Kosowski et al. (2006), Barras et al. (2010), Fama \& French (2010) and Ferson \& Chen (2015) take a frequentist approach and estimate the population distribution by bootstrapping the estimated skill of the funds. Both Chen et al. (2017) and Harvey \& Liu (2018) model the population distribution with a finite mixture of normals and estimate the mixture parameters with an EM algorithm. Barras et al. (2018) use a nonparametric method to estimate the population distribution but do not use the information from the population in the estimation of a fund's skill.

Jones \& Shanken (2005) estimate the population distribution from a parametric Bayesian perspective using a hierarchical normal prior for skill. Others like Pástor \& Stambaugh (2002b) assume a distribution for the population. Baks et al. (2001), Pástor \& Stambaugh (2002a), and Avramov \& Wermers (2006) also assume they know the cross-sectional distribution. Each finds their estimate of skill to be sensitive to the choice of the population distribution.

To our knowledge, no one has estimated mutual fund skill by letting the population distribution be entirely unknown, estimating it, and using it to infer the skill level of the funds. We do this here by modeling the unknown population distribution with a Bayesian, nonparametric, hierarchical prior. This nonparametric prior is an infinite mixture of normals with unknown mixture weights, locations, scales, and mixture order. We infer these mixture unknowns with an unsupervised learning ${ }^{1}$ approach where we partition the panel of mutual funds into a finite number of groups (mixture clusters) where the members of a group all have the same average stock-picking ability and variability (mixture location and scale). ${ }^{2}$

We leverage these random partitions to resolve the uncertainty in the skill level of a member fund by pooling the information of the group's other funds. Sharing the groups information is especially important in resolving the uncertainty around the skill level of newer funds with short performance histories. Partitioning the funds also eliminates the global shrinkage issues that plague parametric hierarchical priors. Extraordinarily skilled funds are allowed to have their own group and not have their estimate of skill shrunk towards

\footnotetext{
${ }^{1}$ See Murphy (2012) for an introduction to unsupervised learning.

${ }^{2}$ Learning about the cross-sectional distribution of skill by partitioning funds into different groups is similar to Cohen et al. (2005) idea of judging a fund by the company it keeps. However, our approach is unsupervised and, hence, does not use any information about a fund beyond its return history.
} 
the global industry average as in Jones \& Shanken (2005).

Using return data from the entire actively managed, US domestic equity fund, industry, we find the population distribution of skill to be fat-tailed, slightly skewed towards better stock-picking ability, and having three modes. These three modes are i) a minor mode where skill is extraordinarily high, ii) a secondary mode where funds lose money for its investors, and iii) a primary mode at a skill level where funds cover the average fees charged investors. As a result of our nonparametric population distribution, there is a greater chance a fund will be extraordinarily skilled relative to a normally distributed population. We also see that the exceptionally skilled and unskilled funds that we uncover with our nonparametric population distribution look rather ordinary under a normal population distribution.

We organize the paper in the following manner. In Section 2 we present a mutual fund investor's investment decision and how he applies Bayes rule to update both his understanding of the population distribution of skill and the potential skill of a fund. Section 3 describes our nonparametric, hierarchical, Dirichlet process mixture, prior for the skill level of the funds and the initial population distribution for this nonparametric prior. We then describe in Section 4 the Bayesian nonparametric learning that comes with the Dirichlet process, followed by the model's Markov Chain Monte Carlo sampler in Section 5. In Section 6 we apply our Bayesian nonparametric learning approach along with a Bayesian parametric hierarchical model and a idiosyncratic Bayesian parametric model to a panel of 5,136 actively managed mutual funds. Section 7 summarizes our findings and provides our conclusions.

\section{Investors decision}

In this section we analyze the population distribution of mutual fund performance from the perspective of rational Bayesian investors who choose between a risk-free asset, a set of benchmark assets, and an array of actively managed mutual funds. Our investors' decision differs from that in Baks et al. (2001) (BMW) where the decision to invest in a specific fund is treated independently from the deliberations around investing in the other funds. Instead, we follow Jones \& Shanken (2005) (JS) and assume the investors choose to invest in a current mutual fund by analyzing the return performance of past and present mutual funds when determining skill and the population distribution.

Following Jensen (1968), the risk-free adjusted, gross returns ${ }^{3}$ for $J$, past, and present,

\footnotetext{
${ }^{3}$ We analyze gross returns because expenses and fees vary across funds and over time, and the management company generally sets them. For example, in the economic model of fund behavior by Berk \& Green (2004), the model predicts the economic rents generated by a skilled fund will be captured by management through
} 
mutual funds, are assumed to follow the linear factor model

$$
r_{i, t}=\alpha_{i}+\beta_{i}^{\prime} F_{t}+\sigma_{i} \epsilon_{i, t}, \quad i=1, \ldots, J, \text { and } t=\tau_{i}, \ldots, T_{i},
$$

where $1 \leq \tau_{i}$, and the length of each series is $\mathcal{T}_{i}=T_{i}-\tau_{i}+1$ such that $\mathcal{T}_{i}$ and $\mathcal{T}_{i^{\prime}}$ do not have to be equal. As an unbalanced panel the starting points, $\tau_{i}, i=1, \ldots, J$, do not need to be same.

Monthly fund returns do not exhibit the non-Gaussian behavior or time-varying variances often seen in high-frequency asset returns. So we assume the innovations are Gaussian white noise, $\epsilon_{i, t} \stackrel{i i d}{\sim} N(0,1)$. We also assume each fund has a stock-picking strategy of its own and does not mimic or borrow from the other fund's approaches. In other words, the return innovations are assumed to be uncorrelated across funds such that $\operatorname{Cov}\left(\epsilon_{i, t}, \epsilon_{i^{\prime}, t}\right)=0 .{ }^{4}$ Under these assumptions the sampling distribution representation of Eq. (1) is

$$
r_{i, t} \mid \alpha_{i}, \beta_{i}, \sigma_{i}^{2} \sim N\left(r_{i, t} \mid \alpha_{i}+\beta_{i}^{\prime} F_{t}, \sigma_{i}^{2}\right)
$$

The vector of passive benchmark returns, $F_{t}$, are observed by our investors at the end of each month $t$. Later, in the empirical application these benchmark returns will consist of the four passive risk factors; the three-factor model of Fama \& French (1993) and the momentum portfolio factor of Carhart (1997). Under these risk factor Eq. (1) becomes

$$
r_{i, t}=\alpha_{i}+\beta_{i, R} \cdot \mathrm{RMRF}_{t}+\beta_{i, S} \cdot \mathrm{SMB}_{t}+\beta_{i, H} \cdot \mathrm{HML}_{t}+\beta_{i, M} \cdot \mathrm{MOM}_{t}+\sigma_{i} \epsilon_{i, t},
$$

where $\mathrm{RMRF}_{t}$ is the excess market return in the $t$ th month, $\mathrm{SMB}_{t}$ and $\mathrm{HML}_{t}$ are the size and book-to-market factors, and $\mathrm{MOM}_{t}$ is the monthly momentum return.

In both Eq. (1) and (2), the parameter $\alpha_{i}$ is assumed to measure fund $i$ 's ability to identify under-priced stocks and is the only parameter measuring this skill. BMW show that a Bayesian, mean-variance, investor will invest in an existing fund if and only if the expected posterior value of $\alpha_{i}$ is greater than zero and at least as large as the fund's fees; i.e., the mutual fund is expected to outperform a costless portfolio comprised of the benchmark returns, $F$, and cover the fund's fees. According to BMW the Bayesian investor also chooses to invest in a new fund if and only if the expected value of alpha over the posterior population distribution is positive and exceeds the average fee charged by the industry. How much the investor invests in either a new or existing fund then depends on the level of uncertainty in the investment as measured by the standard deviation of the relevant posterior.

higher fees.

${ }^{4}$ JS relax the cross-sectional independence assumption and use a hidden factor model, which improved the precision but did not affect the location of the skill estimates. 
In our Bayesian nonparametric approach, an investor's initial guess about the population distribution of skill or a particular fund's level of expertise is represented by the hierarchical prior $\pi(\alpha \mid \theta)$ with the hyperparameter $\theta$. Knowledge about the $i$ th fund's ability, along with an understanding of the population, increases as we observe the risk-adjusted gross returns of any actively managed fund. For instance, if we only see the returns for the $i$ th fund, we directly update our guess about the skill of the $i$ th fund by applying Bayes rule to the posterior

$$
\pi\left(\alpha_{i} \mid r_{i}, \theta\right) \propto \pi\left(\alpha_{i} \mid \theta\right) f\left(r_{i} \mid \alpha_{i}\right)
$$

where

$$
f\left(r_{i} \mid \alpha_{i}\right)=\prod_{t=\tau_{i}}^{T_{i}} N\left(r_{i, t}-\beta_{i}^{\prime} F_{t}, \mid \alpha_{i}, \sigma_{i}^{2}\right),
$$

is the likelihood conditional on $\beta_{i}$ and $\sigma_{i}^{2}$, and $r_{i}=\left(r_{i, \tau_{i}}, \ldots, r_{i, T_{i}}\right)^{\prime} .^{5}$

Alternatively, if the returns are from the $J-1$ other funds, we update our guess about the population distribution with the posterior predictive distribution

$$
\pi\left(\alpha \mid r_{-i}\right)=\int \pi(\alpha \mid \theta) d G\left(\theta \mid r_{-i}\right)
$$

where $r_{-i}$ are the return histories of all the funds besides the $i$ th fund. The $\alpha$ in Eq. (5) represents the skill level of any fund not included in $r_{-i}$. Here we are referring to the $i$ th fund generically such that $\alpha$ could also represent the skill of fund not included in the panel of $J$ funds. In Eq. (5),

$$
G\left(\theta \mid r_{-i}\right) \propto G(\theta) p\left(r_{-i} \mid \theta\right)
$$

is the posterior for the hyperparameter where the connection between $\theta$ and $r_{-i}$ is made via $p\left(r_{-i} \mid \theta\right)=\int f\left(r_{-i} \mid \alpha_{-i}\right) \pi\left(\alpha_{-i} \mid \theta\right) d \alpha_{-i}$.

The posterior predictive distribution in Eq. 5 has updated our initial guess for the population distribution, the prior, $\pi(\alpha)$, to the posterior population distribution, $\pi\left(\alpha \mid r_{-i}\right)$. This "updated prior" gets augmented with the additional information observed in the $i$ th fund's likelihood. Now our assessment of the $i$ th fund's level of skill is found in the fund's posterior

$$
\pi\left(\alpha_{i} \mid r_{i}, r_{-i}\right) \propto \pi\left(\alpha_{i} \mid r_{-i}\right) f\left(r_{i} \mid \alpha_{i}\right)
$$

\footnotetext{
${ }^{5}$ Note we have suppressed $\beta_{i}$ and $\sigma_{i}^{2}$ from conditioning argument of the likelihood to simplify the notation.
} 
Given the cross-sectional information in Eq. (7) of past and present mutual fund performance, the posterior for fund $i$ 's alpha is at least as well informed as $\pi\left(\alpha_{i} \mid r_{i}\right)$ and better if $r_{i}$ is short or non-existent. ${ }^{6}$

Thus far we are only learning about the alphas and $\theta$ conditional on the particular prior distribution $\pi(\alpha, \theta)=\pi(\alpha \mid \theta) G(\theta)$ where the distributions $\pi(\alpha)$ and $G(\theta)$ are assumed to be known. In the following section, we let our beliefs about the cross-sectional distribution of skill be completely flexible, in other words, nonparametric, by letting $G$ be unknown. We then learn about the population distribution of skill by using the information from the panel of returns to update $G$, the alphas, and $\theta$. We now show how one learns about the unknown cross-sectional distribution of skill as one learns about $G$.

\section{Initial beliefs about the population}

We assume the prior beliefs for the distribution of alpha is independent from the risk-factors and return variance by letting $\pi\left(\alpha_{i}, \beta_{i}, \sigma_{i}^{2}\right)=\pi\left(\alpha_{i}\right) \pi\left(\beta_{i}, \sigma_{i}^{2}\right) .{ }^{7}$ We follow Muller \& Rosner (1997) and let the prior for the alphas be the nonparametric, Dirichlet Process mixture, prior (DPM)

$$
\begin{aligned}
\alpha_{i} \mid \mu_{\alpha, i}, \sigma_{\alpha, i}^{2} & \sim N\left(\mu_{\alpha, i}, \sigma_{\alpha, i}^{2}\right), \\
\mu_{\alpha, i}, \sigma_{\alpha, i}^{2} \mid G & \sim G, \\
G \mid G_{0} & \sim D P\left(B, G_{0}\right),
\end{aligned}
$$

where the unknown hyperprior distribution, $G$, is modeled in terms of Ferguson's (1973) Dirichlet process, $D P\left(B, G_{0}\right){ }^{8}$

The DPM has been used extensively in econometrics to model unknown distributions (see Chib \& Hamilton 2002, Hirano 2002, Jensen 2004, Jensen \& Maheu 2010, Bassetti et al. 2014). A primary reason for this is the DP's almost sure discrete representation of the unknown hyperprior distribution

$$
G \stackrel{a s}{=} \sum_{k=1}^{\infty} \omega_{k} \mathbf{1}_{\left\{\mu_{\alpha, k}^{*}, \sigma_{\alpha, k}^{2 *}\right\}},
$$

\footnotetext{
${ }^{6}$ We could also use the cross-section of return histories to update the priors for $\beta_{i}$ and $\sigma_{i}$. However, since our focus is on mutual fund performance we let the priors for $\beta_{i}$ and $\sigma_{i}$ be ex ante uninformative priors; i.e., we assume the betas and sigmas are idiosyncratic over the cross-section of funds. Investigating how to learn about the betas and sigmas would be a worthy research project.

${ }^{7}$ One could assume investors have a joint prior for $\left(\alpha_{i}, \beta_{i}, \sigma_{i}^{2}\right)$. However, learning this distribution would require assigning a fund to a cluster based on all the unknown parameters and not just alpha. Grouping funds by ability would no longer be our objective, so, we assume a separate prior for the alphas, betas and sigmas.

${ }^{8}$ See Kleinman \& Ibrahim (1998), Burr \& Doss (2005), Ohlssen et al. (2007), Dunson (2010) and Chapter 23 of Gelman et al. (2013) and references therein for the mathematical details of the Dirichlet process.
} 
with $\left(\mu_{\alpha, k}^{*}, \sigma_{\alpha, k}^{2 *}\right) \stackrel{i i d}{\sim} G_{0}, \omega_{k}=w_{k} \prod_{k^{\prime}<k}\left(1-w_{k^{\prime}}\right)$, where $w_{k} \sim \operatorname{Beta}(1, B)$, and $\mathbf{1}_{\left\{\mu_{\alpha, k}^{*}, \sigma_{\alpha, k}^{2 *}\right\}}$ is a point mass at $\left(\mu_{\alpha, k}^{*}, \sigma_{\alpha, k}^{2 *}\right)$. This discreteness leads to the partitioning of the alphas into groups.

Another important reason for using the DP is its ease of use. As a conjugate distribution, the DP lends itself to the simple, and efficient, sampling algorithm of West et al. (1994). ${ }^{9}$ Draws from this sampler are made with distributions that are known, and the draws quickly converge to realizations from the posterior distribution of the nonparametric hierarchical prior.

The arguments to the DP distribution are the base distribution, $G_{0}$, and the concentration parameter, $B$. The expectation of the DP is $E[G]=G_{0}$, so, the base distribution, $G_{0}$, represents our prior knowledge about $G$, and indirectly, the population distribution. The concentration parameter, $B$, is a positive scalar. Since $\operatorname{Var}[G] \equiv\left[G_{0}\left(1-G_{0}\right)\right] /(1+B), B$ can be thought of as the inverse variance of $G$. The larger $B$ is the more confident we are about $G_{0}$ being the hyperprior $G$. In the limit, $G \rightarrow G_{0}$, and $\omega_{k} \rightarrow 0$, as $B \rightarrow \infty .{ }^{10}$ In our empirical application $B$ is unknown and estimated.

One needs to be thoughtful about choosing $G_{0}$ since it plays an important role in how open-minded we are about mutual fund skill. For example, if we were certain about the average skill and the variance of the population we might choose a base distribution of $G_{0} \equiv$ $\mathbf{1}_{\left\{m_{0}, s_{0}^{2}\right\}}\left(\mu_{\alpha}, \sigma_{\alpha}^{2}\right)$, where $m_{0}$ and $s_{0}^{2}$ are set to prespecified values. Given the degenerative nature of this base distribution our initial guess for the cross-sectional distribution of the alphas is

$$
\begin{aligned}
\widehat{\pi}_{\mathbf{1}_{\left\{m_{0}, s_{0}^{2}\right\}}(\alpha)} & \equiv E_{G}\left[\int N\left(\alpha \mid \mu_{\alpha}, \sigma_{\alpha}^{2}\right) d G\left(\mu_{\alpha}, \sigma_{\alpha}^{2}\right)\right] \\
& =\int N\left(\alpha \mid \mu_{\alpha}, \sigma_{\alpha}^{2}\right) d G_{0}\left(\mu_{\alpha}, \sigma_{\alpha}^{2}\right), \\
& =\int N\left(\alpha \mid \mu_{\alpha}, \sigma_{\alpha}^{2}\right) \mathbf{1}_{\left\{m_{0}, s_{0}^{2}\right\}}\left(\mu_{\alpha}, \sigma_{\alpha}^{2}\right) d\left(\mu_{\alpha}, \sigma_{\alpha}^{2}\right), \\
& =N\left(\alpha \mid m_{0}, s_{0}^{2}\right) .
\end{aligned}
$$

For those whose prior is $\widehat{\pi}_{\mathbf{1}_{\left\{m_{0}, s_{0}^{2}\right\}}}(\alpha)$, they believe they know the population to be normally distributed with a mean and variance equal to $m_{0}$ and $s_{0}^{2}$, respectively. BMW, Pástor \& Stambaugh (2002a), and Pástor \& Stambaugh (2002b) either implicitly or explicitly assume such strong prior beliefs about the population. For instance, any empirical study of

\footnotetext{
${ }^{9}$ If we were concerned about the computing time involved in re-estimating the nonparametric, posterior, population distribution as new return data becomes available we could compute in real time the posterior population distribution using the particle learning, sequential sampler of Carvalho et al. (2010).

${ }^{10} \mathrm{~B}$ plays an important role in the creation of clusters as the number of funds grows. We will explain this when we present the clustering properties of the DP prior.
} 
mutual fund skill where ordinary least square estimates of the alphas are used implicitly sets $m_{0}=0$ and $1 / s_{0}^{2}=0$. As a result the prior predictive distribution $\widehat{\pi}_{\mathbf{1}_{\{0, \infty\}}}(\alpha) \propto C$ says there is no information to be found in the cross-section. Instead, each fund's level of skill is idiosyncratic to the fund.

If we are sure about $G_{0}$ being the unknown hyperprior, $G$, then, $B \rightarrow \infty$, and we would only need to learn about $\mu_{\alpha}$ and $\sigma_{\alpha}^{2}$. Suppose we were confident the Normal, InverseGamma, base distribution was the hyperprior, then

$$
G \rightarrow N I G\left(m_{0}, \sigma_{\alpha}^{2} / \kappa_{0}, \nu_{0} / 2, s_{0}^{2}, \nu_{0} / 2\right), \text { as } B \rightarrow \infty
$$

where $m_{0}$ and $\sigma_{\alpha}^{2} / \kappa_{0}$ are the mean and variance to the conditional Normal distribution for $\mu_{\alpha}$, and $\nu_{0} / 2$ and $s_{0}^{2} \nu_{0} / 2$ are respectively the scale and shape of the Inverse-Gamma distribution for $\sigma_{\alpha}^{2}$.

According to Bernardo \& Smith (2000, Appendix A2), such prior beliefs about the hyperprior are those where the prior predictive distribution is the Student-t distribution

$$
\begin{aligned}
\pi_{N I G}(\alpha) & =\int N\left(\alpha \mid \mu_{\alpha}, \sigma_{\alpha}^{2}\right) N I G\left(\mu_{\alpha}, \sigma_{\alpha}^{2} \mid m_{0}, \sigma_{\alpha}^{2} / \kappa_{0}, \nu_{0} / 2, s_{0}^{2} \nu_{0} / 2\right) d\left(\mu_{\alpha}, \sigma_{\alpha}^{2}\right) \\
& =t_{\nu_{0}}\left(\alpha \mid m_{0},\left(\frac{\kappa_{0}+1}{\kappa_{0}}\right) \nu_{0} s_{0}^{2}\right)
\end{aligned}
$$

with $\nu_{0}$ degrees of freedom, a mean of $m_{0}$, and scale $\sqrt{\left[\left(\kappa_{0}+1\right) / \kappa_{0}\right] \nu_{0} s_{0}^{2}}$.

Because we are so sure of $G_{0}$ being $G$ when $B \rightarrow \infty, \pi_{N I G}(\alpha)$ is also the posterior predictive distribution. Hence, we are so confident in our initial guess for the population distribution there is nothing in the cross-section of fund return data that can convince us otherwise.

In this paper, we choose to learn everything about the population distribution from the cross-section of fund returns. To do this, our prior knowledge of the hyperprior is

$$
G_{0} \equiv N I G\left(m_{0}, \sigma_{\alpha}^{2} / \kappa_{0}, \nu_{0} / 2, s_{0}^{2}, \nu_{0} / 2\right)
$$

with $m_{0}=0, \kappa_{0}=0.1, \nu_{0}=0.01$ and $s_{0}^{2}=0.01$. The Student-t, prior predictive

$$
\begin{aligned}
\widehat{\pi}_{N I G}(\alpha) & =\int N\left(\alpha \mid \mu_{\alpha}, \sigma_{\alpha}^{2}\right) E\left[d G\left(\mu_{\alpha}, \sigma_{\alpha}^{2}\right)\right] \\
& =t_{\nu_{0}}\left(\alpha \mid m_{0},\left(\frac{\kappa_{0}+1}{\kappa_{0}}\right) \nu_{0} s_{0}^{2}\right)
\end{aligned}
$$

is then proper, but diffuse.

Given these arguments for Eq. (19), we initially think the average fund does not have the skill to beat a passive portfolio $\left(m_{0}=0\right)$. Furthermore, because we set $\nu_{0}$ equal to 0.01 , 
we initially believe that there is so much variability in mutual fund skill that the population variance does not exist. We now explain how we learn about $G$, and hence, learn about the population distribution $\pi(\alpha)$.

\section{Bayesian nonparametric learning}

The next step is learning about the hyperprior distribution, G. Suppose, hypothetically, that we see a realization of the hyperparameters, $\left(\mu_{\alpha, 1}, \sigma_{\alpha, 1}^{2}\right) \sim G$, where $\mu_{\alpha, 1}$ is the average skill level of a fund and $\sigma_{\alpha, 1}^{2}$ is the variance in the fund's skill level. Being a realization from $G$, we use $\mu_{\alpha, 1}$, and $\sigma_{\alpha, 1}^{2}$, to update our understanding of $G$ with the posterior DP

$$
G \mid \mu_{\alpha, 1}, \sigma_{\alpha, 1}^{2} \sim D P\left(1+B, G_{1}\right)
$$

where the updated base distribution is

$$
G_{1} \equiv \frac{B}{1+B} G_{0}+\frac{1}{1+B} \mathbf{1}_{\left\{\mu_{\alpha, 1}, \sigma_{\alpha, 1}^{2}\right\}}
$$

(see Blackwell \& MacQueen (1973) who prove that this conjugacy property holds for the DP).

In Eq. (20) the concentration parameter has increased to $1+B$, so we are a bit more confident in the updated base distribution, $G_{1}$, representing $G$. This new guess for $G$ consists of a mixture of our original guess, $G_{0}$, and the information contained in the empirical

distribution, $\mathbf{1}_{\left\{\mu_{\alpha, 1}, \sigma_{\alpha, 1}^{2}\right\}}$. Given $G_{1}$, our guess for the population distribution is now the posterior predictive distribution

$$
\begin{aligned}
\widehat{\pi}_{N I G}\left(\alpha \mid \mu_{\alpha, 1}, \sigma_{\alpha, 1}^{2}\right) & =\int N\left(\alpha \mid \mu_{\alpha}, \sigma_{\alpha}^{2}\right) d G_{1}\left(\mu_{\alpha}, \sigma_{\alpha}^{2}\right), \\
& =\frac{B}{1+B} t_{\nu_{0}}\left(\alpha \mid m_{0},\left(\frac{\kappa_{0}+1}{\kappa_{0}}\right) \nu_{0} s_{0}^{2}\right)+\frac{1}{1+B} N\left(\alpha \mid \mu_{\alpha, 1}, \sigma_{\alpha, 1}^{2}\right),
\end{aligned}
$$

where the mixture weight, $1 /(1+B)$, is the probability we assign to a mutual fund, we do not have information on, belonging to the group whose average level of skill is $\mu_{\alpha, 1}$, and whose variability in skill is $\sigma_{\alpha, 1}^{2}$.

It also follows from Eq. (22) that we assign a $B /(1+B)$ chance to the same fund we know nothing about belonging to a new group whose average and variance in skill is different from $\mu_{\alpha, 1}$ and $\sigma_{\alpha, 1}^{2}$. Assigning funds to a new group gives us the flexibility to continue to increase the number of mixture clusters as the number of mutual funds in our panel grows; i.e., the DP has an infinite number of mixture clusters available for new funds to be assigned to. 
We continue to apply this unsupervised probabilistic approach to categorizing funds as we hypothetically observe realizations from $G$ for the $J$ mutual funds. After "seeing" $\mu_{\alpha, i}$, and $\sigma_{\alpha, i}^{2}, i=1, \ldots, J$, our posterior $\mathrm{DP}$ for $G$ is

$$
G \mid \mu_{\alpha, 1}, \sigma_{\alpha, 1}^{2}, \ldots, \mu_{\alpha, J}, \sigma_{\alpha, J}^{2} \sim D P\left(J+B, G_{J}\right)
$$

where

$$
G_{J} \equiv \frac{B}{J+B} G_{0}+\sum_{k=1}^{K} \frac{n_{k}}{J+B} \mathbf{1}_{\left\{\mu_{\alpha, k}^{*}, \sigma_{\alpha, k}^{2 *}\right\}},
$$

is our guess for $G$.

In Eq. (24), the updated base distribution, $G_{J}$, shows how our Bayesian nonparametric method of learning has uncovered $K \leq J$ groups each with its own unique mean and variance, $\mu_{\alpha, k}^{*}$, and $\sigma_{\alpha, k}^{2 *}$, for $k=1, \ldots, K$. These $K$ groups each contain $n_{k}, k=1, \ldots, K$, funds such that $\sum_{k} n_{k}=J$.

The concentration parameter in Eq. (23) has increased to $J+B$, so our confidence in the guess for $G$ has grown as has our confidence in the estimate for the population distribution

$$
\begin{aligned}
\widehat{\pi}_{N I G}\left(\alpha \mid \mu_{\alpha, 1}, \sigma_{\alpha, 1}^{2}, \ldots, \mu_{\alpha, J}, \sigma_{\alpha, J}^{2}\right)= & \frac{B}{J+B} t_{\nu_{0}}\left(\alpha \mid m_{0},\left(\frac{\kappa_{0}+1}{\kappa_{0}}\right) \nu_{0} s_{0}^{2}\right) \\
& +\sum_{k=1}^{K} \frac{n_{k}}{J+B} N\left(\alpha \mid \mu_{\alpha, k}^{*}, \sigma_{\alpha, k}^{2 *}\right) .
\end{aligned}
$$

When we know nothing about a fund, according to Eq. (25), the probability of assigning the fund to one of the $K$ groups depends on the group's size, $n_{k}$. In our mind, larger groups have a greater chance of having a new fund assigned to it. However, the number of groups also depends on how confident we are in our initial guess $G_{0}$; i.e., the concentration parameter $B$. The larger $B$ is the more groups we will partition the cross-section of mutual funds in to.

We are thus learning about the population distribution of mutual fund skill by assuming very little about the cross-sectional distribution of skill, but then learning about it by flexibly forming a mixture of normals where the number of clusters are identified by partitioning the funds into groups having the same average skill and variability. Our nonparametric approach allows the number of groups to grow with the size of the cross-section and will allow a wider spectrum of skill especially extraordinarily skilled and unskilled funds.

\section{Inference}

To resolve the uncertainty around the alphas, the number of mixture clusters, the clusters' averages and variances, the concentration parameter, and the population distribution of 
mutual fund skill, we combine fund-level return data with our initial beliefs to form a posterior for these unknowns. The joint posterior distribution for these unknowns, however, is very complex and does not have a known analytical distribution. Analysis of the complex joint posteriors requires judiciously breaking it up into its conditional posteriors and using a Markov Chain Monte Carlo sampler to make joint posterior draws by sequentially sampling from the conditional posteriors.

The conditionals we sample from are structured by the hierarchical form of our nonparametric model. Let $s=\left(s_{1}, \ldots, s_{J}\right)$ be a $J$ length vector containing all the funds group assignments $s_{i}$ where $s_{i}=k$ when $\left(\mu_{\alpha, i}, \sigma_{\alpha, i}^{2}\right)=\left(\mu_{\alpha, k}^{*}, \sigma_{\alpha, k}^{2 *}\right)$. The sequence of conditional posterior distribution can then be sampled by:

1. Drawing $\beta_{i}$ and $\sigma_{i}^{2}$ conditional on $r_{i}$, and $\alpha_{i}$, for $i=1, \ldots, J$,

2. Drawing $\alpha_{i}$ conditional on $r_{i}, \beta_{i}, \sigma_{i}^{2}$ and $\left(\mu_{\alpha, s_{i}}^{*}, \sigma_{\alpha, s_{i}}^{2 *}\right)$, for $i=1, \ldots, J$,

3. Drawing $s, K,\left(\mu_{\alpha, k}^{*}, \sigma_{\alpha, k}^{2 *}\right), k=1, \ldots, K$, conditional on $\alpha_{1}, \ldots, \alpha_{J}$,

4. Drawing $B$ conditional on $K$.

In Step 1, our prior knowledge for the factor loading vector, $\beta_{i}$, and the return variance, $\sigma_{i}^{2}$, is represented by the Jeffreys prior

$$
\pi\left(\beta_{i}, \sigma_{i}^{2}\right) \propto 1 / \sigma_{i}^{2}
$$

Under this prior, the conditional posterior for $\beta_{i}$ in Step 1 depends only on the returnbased information, $r_{i}$. The conditional $p\left(\beta_{i} \mid r_{i}, \alpha_{i}, \sigma_{i}^{2}\right)$ is a normally distributed conditional posterior with mean and covariance equal to the least squares regression estimator of the dependent variable $r_{i t}-\alpha_{i}$ projected onto the explanatory variables $F_{i t}, t=\tau_{i}, \ldots, T_{i}$. The marginal conditional posterior distribution $p\left(\sigma_{i}^{2} \mid r_{i}, \alpha_{i}, \beta_{i}\right)$ is a Inverse-Gamma distribution with scale, $\mathcal{T}_{i}-4$, and shape equal to the sum of squared error from the above linear least squares regression divided by the scale, $\mathcal{T}_{i}-4$.

In Step 2, the prior for $\alpha_{i}$ is the cross-sectional distribution of the $s_{i}$ th group,

$$
\alpha_{i} \mid \mu_{\alpha, s_{i}}^{*}, \sigma_{\alpha, s_{i}}^{2 *} \sim N\left(\mu_{\alpha, s_{i}}^{*}, \sigma_{\alpha, s_{i}}^{* 2}\right) .
$$

Combining this cross-sectional information with the likelihood from the $i$ th fund's return history, $r_{i}$, the conditional posterior in Step 2 is

$$
\alpha_{i} \mid r_{i}, \beta_{i}, \sigma_{i}^{2}, \mu_{\alpha, s_{i}}^{*}, \sigma_{\alpha, s_{i}}^{2 *} \sim N\left(a_{i}, b_{i}\right),
$$


where the posterior mean is

$$
a_{i}=\left(\frac{\mu_{\alpha, s_{i}}^{*}}{\sigma_{\alpha, s_{i}}^{2 *}}+\sum_{t=\tau_{i}}^{T_{i}} r_{i, t}^{*}\right) /\left(\frac{1}{\sigma_{\alpha, s_{i}}^{2 *}}+\mathcal{T}_{i}\right),
$$

with

$$
r_{i, t}^{*} \equiv\left(r_{i, t}-\beta_{i}^{\prime} F_{i, t}\right) / \sigma_{i}
$$

being the risk adjusted return, and the posterior variance is

$$
b_{i}=\left(1 / \sigma_{\alpha, s_{i}}^{2 *}+\mathcal{T}_{i}\right)^{-1} .
$$

We can think of the sampling in Step 3 as an answer to the question proposed by JS and adapted to our case - when would investors discard the information found in the average skill and variability of the $K$ sub-populations, $\mu_{\alpha, k}^{*}$, and $\sigma_{\alpha, k}^{*}, k=1, \ldots, K$ ? Answering this question for each fund amounts to drawing the assignment vector $s$ by sequentially drawing each fund's $s_{i}$ according to the probabilities

$$
\begin{aligned}
P\left(s_{i}=k\right) & =\frac{n_{k}^{(-i)}}{B+J-1} f_{N}\left(\alpha_{i} \mid \mu_{\alpha, k}^{*}, \sigma_{\alpha, k}^{* 2}\right), \quad k=1, \ldots, K^{(-i)}, \\
P\left(s_{i}=K^{(-i)}+1\right) & =\frac{B}{B+J-1} f_{t}\left(\alpha_{i} \mid m_{0},\left(\frac{\kappa_{0}+1}{\kappa_{0}}\right) \nu_{0} s_{0}^{2}\right),
\end{aligned}
$$

where, after the $i$ th fund has been excluded from the sample, $n_{k}^{(-i)}$ is the number of funds belonging to the $k$ th group, and $K^{(-i)}$ is the total number of clusters. $K^{(-i)}$ will equal $K-1$ if the $i$ th funds is the only member of its group. Otherwise, $K^{(-i)}$ equals $K$.

Eq. (32) is the probability we discard the information contain in the performance of the other funds and only rely on the fund's performance history to determine the funds alpha. The odds of this occurring are a function of the concentration parameter, $B$, and the value of the prior predictive distribution evaluated at the draw of $\alpha_{i}$.

After we assign every fund to a group, and in the process determine the total number of mixture clusters, $K$, we pool together the alphas from those funds belonging to the same group and form our posterior beliefs about the average skill level and variability of the cluster by drawing $\mu_{\alpha, k}^{*}$ and $\sigma_{\alpha, k}^{2 *}$. Given the DP base distribution, $G_{0} \equiv$ $N I G\left(m_{0}, \sigma_{\alpha}^{2} / \kappa_{0}, \nu_{0} / 2, s_{0}^{2} \nu_{0} / 2\right)$, from Section 3 , draws of $\sigma_{\alpha, k}^{2 *}$, are from

$$
\begin{aligned}
& \sigma_{\alpha, k}^{2 *} \mid\left\{\alpha_{i}\right\}_{i: s_{i}=k} \sim N I G\left(\frac{\nu_{0}+n_{k}}{2},\right. \\
&\left.\frac{1}{2}\left[\nu_{0} s_{0}^{2}+\sum_{i: s_{i}=k}\left(\alpha_{i}-\bar{\alpha}_{k}\right)^{2}+\frac{n_{k} \kappa_{0}}{\kappa_{0}+n_{k}}\left(m_{0}-\bar{\alpha}_{k}\right)^{2}\right]\right),
\end{aligned}
$$


where $\bar{\alpha}_{k}=n_{k}^{-1} \sum_{i: s_{i}=k} \alpha_{i}$. Draws of $\mu_{\alpha, k}^{*}$ are then made from

$$
\mu_{\alpha, k}^{*} \mid\left\{\alpha_{i}\right\}_{i: s_{i}=k}, \sigma_{\alpha, k}^{2 *} \sim N\left(\frac{\kappa_{0} m_{0}+n_{k} \bar{\alpha}_{k}}{\kappa_{0}+n_{k}}, \frac{\sigma_{k}^{2 *}}{\kappa_{0}+n_{k}}\right) .
$$

Lastly, in Step 4 we draw the concentration parameter $B$ from $\pi(B \mid K)$ using the sampler described in Appendix A.5 of Escobar \& West (1995).

Later in the empirical application, we initialize our sampler by setting all the funds' alphas equal to zero. The concentration parameter, $B$, is initialized with a random draw from its prior, $\pi(B) \equiv \operatorname{Gamma}(2.0,30.0)$. This draw of $B$, along with the Normal, InverseGamma, base distribution, $G_{0}$, in Eq. (18), are used to initialize $s, K$, and $\left\{\mu_{\alpha, k}^{*}, \sigma_{\alpha, k}^{2 *}\right\}_{k=1, \ldots, K}$, by making $J$ random draws from $D P\left(B, G_{0}\right)$. Given these initial values we can then begin to iterate over the sampler by drawing the $\beta_{i}$ s and $\sigma_{i}^{2} \mathrm{~s}$ in Step 1.

After a burn-in of the sampler where the draws from the conditional posteriors are thrown away to allow the sampler to converge to the posterior distribution, subsequent draws are kept and treated as random realizations from the joint posterior distribution. This randomness in the sampled alphas represents our beliefs about the skill level for each of the funds. We choose to iterate the sampler 40,000 times, keeping the last 30,000 draws of the unknowns to infer all the posteriors.

\subsection{Nesting of approaches}

We point out that the conditional posterior distribution draw of $\alpha_{i}$ in Eq. (27) does not depend on the performance history of other funds. In other words, the guess for each fund's alpha is independent of the other funds. However, the skill of the other funds does influence our guess for $\alpha_{i}$ through the average level of skill, $\mu_{\alpha, s_{i}}^{*}$, and the variance, $\sigma_{\alpha, s_{i}}^{*}$, of the $s_{i}$ th group.

This cluster information is especially important for a fund with short performance window, in other words, when $\mathcal{T}_{i}$ is small, or for a fund with a noisy performance record where $\sigma_{i}^{2}$ is large. Traditional measures of alpha for funds with limited histories are noisy and uncertain (see Kothari \& Warner 2001). But from Eq. (28) we see that the average skill of the $s_{i}$ th group, $\mu_{\alpha, s_{i}}^{*}$, the length of the funds return history, $\mathcal{T}_{i}$, and the noise in the fund's returns, $\sigma_{i}^{2}$, affect the average conditional draw of $\alpha_{i}$. Hence, our nonparametric estimator of fund's alpha will depend more on the average performance of a fund's group, and less on the fund's own performance history, when the fund has a noisy or short performance history. ${ }^{11}$

\footnotetext{
${ }^{11}$ This feature of our Bayesian nonparametric approach would be very helpful measuring the skill level of self-reporting hedge funds where there is no regulation requiring them to report their performance.
} 
The conditional mean of alpha in Eq. (28) also shows how our Bayesian nonparametric approach uses the cross-sectional information differently from JS. In JS there is but one cluster $(K=1)$; all the funds belong to the same group whose average is the industrywide average, $\mu_{\alpha}^{*}$ and whose variability, $\sigma_{\alpha}^{2 *}$, is the industry-wide variance in skill. While insightful in their use of cross-sectional information, we will see in Section 6 that by not allowing for multiple clusters the parametric approach of JS generally under-predict the alphas of skilled funds and over-predict the alphas of unskilled funds. Over and underprediction of skill occurs because the JS guess for $\alpha_{i}$ shrinks towards the average ability of the entire population. ${ }^{12}$

In terms of our Bayesian nonparametric approach, this shrinkage towards the population average by JS is equivalent to the econometrician thinking there is only one group among the mutual funds. One group will be found if the concentration parameter of the DP prior is $B=0$. When $B=0$, the $\omega_{1}=1$ in Eq. (11)'s finite representation of $G$. Hence, the base distribution, $G_{1}$, in the updated DP of Eq. (20) consists of only one cluster, $\left(\mu_{\alpha}^{*}, \sigma_{\alpha}^{2 *}\right)=$ $\left(\mu_{\alpha, 1}, \sigma_{\alpha, 1}^{2}\right)$, where $\left(\mu_{\alpha, 1}, \sigma_{\alpha, 1}^{2}\right) \sim G_{0}$. Each $\left(\mu_{\alpha, i}, \sigma_{\alpha, i}^{2}\right), i=2, \ldots, J$, is a realization from the degenerative base distribution $\mathbf{1}_{\left\{\mu_{\alpha, 1}, \sigma_{\alpha, 1}^{2}\right\}}$, so that the posterior base distribution for $G \mid \mu_{\alpha, 1}, \sigma_{\alpha, 1}^{2}, \ldots, \mu_{\alpha, J}, \sigma_{\alpha, J}^{2}$ is

$$
G_{J}=\frac{J}{J+B} \mathbf{1}_{\left\{\mu_{\alpha}^{*}, \sigma_{\alpha}^{2 *}\right\}}
$$

Step $1 \& 2$ of our sampler remain the same, but Step 3 now only involves drawing $\sigma_{\alpha}^{2 *}$ and $\mu_{\alpha}^{*}$ from Eq. (33) and (34), respectively. ${ }^{13}$

At the other extreme is when $B \rightarrow \infty$. According to the updated base distribution $G_{J}$ in Eq. (24), when $B \rightarrow \infty$, every fund's hyperparameter $\left(\mu_{\alpha, i}, \sigma_{\alpha, i}^{2}\right), i=1, \ldots, J$, is seen as a new independent draw from the initial base distribution, $G_{0}$. Since each realization of the hyperparameter is idiosyncratic we would not partition funds into groups, nor would we learn across the population about the hyperparameters since we would ignore what we learn about the skill of a fund when looking at other funds. In this case, Step 2 of our sampler consists of $J$ independent draws of the alphas where the priors are the idiosyncratic prior distributions, $N\left(\mu_{\alpha, i}, \sigma_{\alpha, i}^{2}\right), i=1, \ldots, J$.

Eq. (27)-(30) also shows how our guess of an extraordinary fund's alpha is no different from the opinion of someone else who chooses to treat such highly skilled funds idiosyncratically. With our Bayesian nonparametric learning approach, highly skilled funds have

\footnotetext{
${ }^{12}$ It is well known that the normal hierarchical prior can lead to poor estimates of the population distribution and the unknown parameter (see Verbeke \& Lesaffre 1996).

${ }^{13}$ This is equivalent to the sampler found in JS.
} 
few peers, and, hence, belong to small groups. In the extreme, a fund that is so highly skilled will have no peers so that $n_{s_{i}}=1$ and $\sigma_{\alpha, s_{i}}^{*}$ will be infinite. In such situations our Bayesian nonparametric approach does not borrow information from the cross-section when guessing the fund's alpha. Instead, our approach treats this extraordinary fund separately from the other funds and according to Eq. (28) draws the fund's conditional posterior alpha from a normal distribution whose first and second moments are those of a ordinary least square estimator of alpha. We will see in the empirical investigation of Section 6.5 that there is only a slight difference between our nonparametric estimate of an extraordinary fund's alpha and the fund's ordinary least square estimate of alpha.

\subsection{Posterior cross-sectional distribution}

In Eq. (25) our best guess for the cross-sectional distribution of the alphas depends on having hypothetically observed the means and variances of the clusters. After observing the return histories from a cross-section of funds we can account for the uncertainty in these unknown mixture means and variances by Rao-Blackwellizing the conditional posterior predictive distribution over the posterior draws of the unknown parameters

$$
\begin{aligned}
\widehat{\pi}_{D P M}\left(\alpha \mid r_{1}, \ldots, r_{J}\right) \approx & M^{-1} \sum_{l=1}^{M}\left[\frac{B^{(l)}}{J+B^{(l)}} t_{\nu_{0}}\left(\alpha \mid m_{0},\left(\frac{\kappa_{0}+1}{\kappa_{0}}\right) \nu_{0} s_{0}^{2}\right)\right. \\
& \left.+\sum_{k=1}^{K^{(l)}} \frac{n_{k}^{(l)}}{J+B^{(l)}} N\left(\alpha \mid \mu_{\alpha, k}^{*(l)}, \sigma_{\alpha, k}^{2 *(l)}\right)\right]
\end{aligned}
$$

where $\left(\mu_{\alpha, k}^{*(l)}, \sigma_{\alpha, k}^{* 2(l)}\right), k=1, \ldots, K^{(l)}$, is the $l$ th draw from the conditional posterior distribution in Step 3 of our sampling algorithm, and $n_{k}^{(l)}, k=1, \ldots, K^{(l)}$, come from the information contained in the $l$ th draw of $s^{(l)}$. Lastly, $B^{(l)}$ is the $l$ th draw from Step 4 of the sampler. This posterior cross-sectional distribution calculation takes into consideration all the uncertainty about the unknowns, including the unknown hyperprior distribution, $G$, by averaging over the posteriors of all the unknowns.

\section{Empirical investigation}

Our empirical application consists of applying the proposed Bayesian nonparametric learning approach to the alphas of the actively managed mutual funds in the data set of Jones \& Shanken (2005). ${ }^{14}$ This data set is comprised of the monthly, gross returns of mutual

\footnotetext{
${ }^{14}$ We would like to thank Chris Jones for graciously providing us with their data.
} 
funds from January 1961 to June 2001. It is a panel with a total of 396,820 monthly observations from 5,136 domestic equity funds. ${ }^{15}$ Like Baks et al. (2001), Jones \& Shanken (2005) and Cohen et al. (2005), we are interested in before cost performance unaffected by the dynamics of the funds' fee schedules so fees and expenses have been added back into the net returns reported in CRSP Mutual Funds data files. Each fund has at least a years worth of return data and the funds have on average 77.3 monthly returns. We include all actively managed, domestic, equity funds in our panel, even the 1,292 funds that were no longer open for business at the end of the sample to avoid any survivorship bias.

\subsection{Posterior number of clusters}

For our Bayesian nonparametric approach where the population distribution of the alphas are modeled with the nonparametric, hierarchical prior of Eq. (8)-(10), we find the posterior median $K$ to be four, with a minimum posterior draw of three, and a maximum of six clusters. The $95 \%$ highest posterior probability density (HPD) interval for $K$ is three to five clusters. Hence, the 5,136 funds are randomly partitioned over a small number of the infinite possible mixture clusters.

The posterior mean for the concentration parameter is 0.1245 with a $95 \%$ HPD interval of $(0.004,0.261)$. Being this close close to zero the posterior for $B$ supports our earlier claims of mutual fund skill not being idiosyncratic to a fund, nor is it normally distributed over the population. Instead, the population distribution of skill is represented by a mixture over a small number of normal distributions.

It is important then to have flexible posterior beliefs about the cross-sectional distribution of mutual fund performance in order to learn about the skill level of a particular fund. Bayesian nonparametric learning gives us the flexibility to increase the number of clusters as the number of the funds grow, to create partitions where the extraordinarily skilled are grouped together, and ordinary funds are in a separate group.

\subsection{Cross-sectional distribution}

In Figure 1 the red line is the density for the posterior cross-sectional distribution of alpha where we learn how skill is distributed over mutual funds by computing the $\widehat{\pi}_{D P M}\left(\alpha \mid r_{1}, \ldots, r_{J}\right)$ in Eq. (35). The blue density is the posterior cross-sectional distribution where skill is believed to be normally distributed whose unknown mean and variance are modeled with the uninformative Jeffreys prior, $\pi\left(\mu_{\alpha}, \sigma_{\alpha}^{2}\right) \propto 1 / \sigma_{\alpha}^{2}$. The uncertainty around the mean and

\footnotetext{
${ }^{15}$ Funds were eliminated that made substantial investments in other asset classes.
} 
variance is integrated out of the normal population distribution with approximation

$$
\widehat{\pi}_{J S}\left(\alpha \mid r_{1}, \ldots, r_{J}\right) \approx M^{-1} \sum_{l=1}^{M} N\left(\alpha \mid \mu_{\alpha}^{(l)}, \sigma_{\alpha}^{2(l)}\right),
$$

where $\left(\mu^{(l)}, \sigma^{2(l)}\right) \sim \pi\left(\mu_{\alpha}, \sigma_{\alpha}^{2} \mid r_{1}, \ldots, r_{J}\right)$, for $l=1, \ldots, M .{ }^{16}$

Very different conclusions about the cross-sectional performance of mutual funds are drawn from the two predictive densities in Figure 1. Our Bayesian, nonparametric, estimator of the population distribution finds three modes. In contrast, the JS population distribution by definition has only one mode. The primary mode for the nonparametric population distribution is $1.8 \%$, and the JS mode is $1.5 \%$. Such alphas just offset the fees charged by an average mutual fund. ${ }^{17}$ An investor who applied the JS approach, thus, believe that, on average, a fund for which they have no information about will just break even. Whereas with our Bayesian nonparametric approach we find that there is a seventy-three percent chance the unknown fund will have an alpha between $0.4 \%$ and $4.0 \%$.

The secondary mode for the nonparametric population distribution in Figure 1 is located at $-0.65 \%$. This negative mode suggests there is are actively managed funds whose average stock-picking ability is detrimental to what an investor could earn on a passively managed fund. ${ }^{18}$ Given the information in this second mode we find that there is a $22 \%$ chance that a fund, for which we have no information about, would produce an alpha between $-5 \%$ to $0.4 \%$ a year.

The third mode of the nonparametric population distribution is a diffuse, low probability, mode located at an alpha of $6 \%$. Although this mode is hard to see in Figure 1, it, along with the population's fat right-hand tail, is easier to detect in the log-scale density plot of Figure 2. There is a $3 \%$ chance a brand new fund, or a fund we do not know, will have the skill to produce an alpha of four to ten percent. On the other hand, such funds have less than a half a percent chance of its alpha being between $-4 \%$ and $-10 \%$. Hence, it is more likely we will find a highly skilled fund than an unskilled fund.

\footnotetext{
${ }^{16}$ OLS alphas in Section 6.5 are idiosyncratic to the fund and, hence, their population distribution is uniform over the real line..

${ }^{17}$ Chen \& Pennacchi (2009) report the average mutual fund's expense fee is 1.14 percent, whereas Berk \& Green (2004) choose a slightly higher management fee of 1.5 percent to account for costs not included in the fee when parameterizing their mutual fund model. We perform our analysis with the larger fee of 1.5 percent to compensate for missing trading costs. Wermers (2011) from ICI estimates actively managed mandates expenses and transactions costs of mutual funds and hedge funds amount to at least two percent a year.

${ }^{18}$ Theoretically, rational investors would pull their money from under-performing funds, causing these unskilled funds to go out of business. Negative alphas leave open the door that some investors act irrationally (see Gruber (1996)), or that investors tolerate short term poor performance.
} 


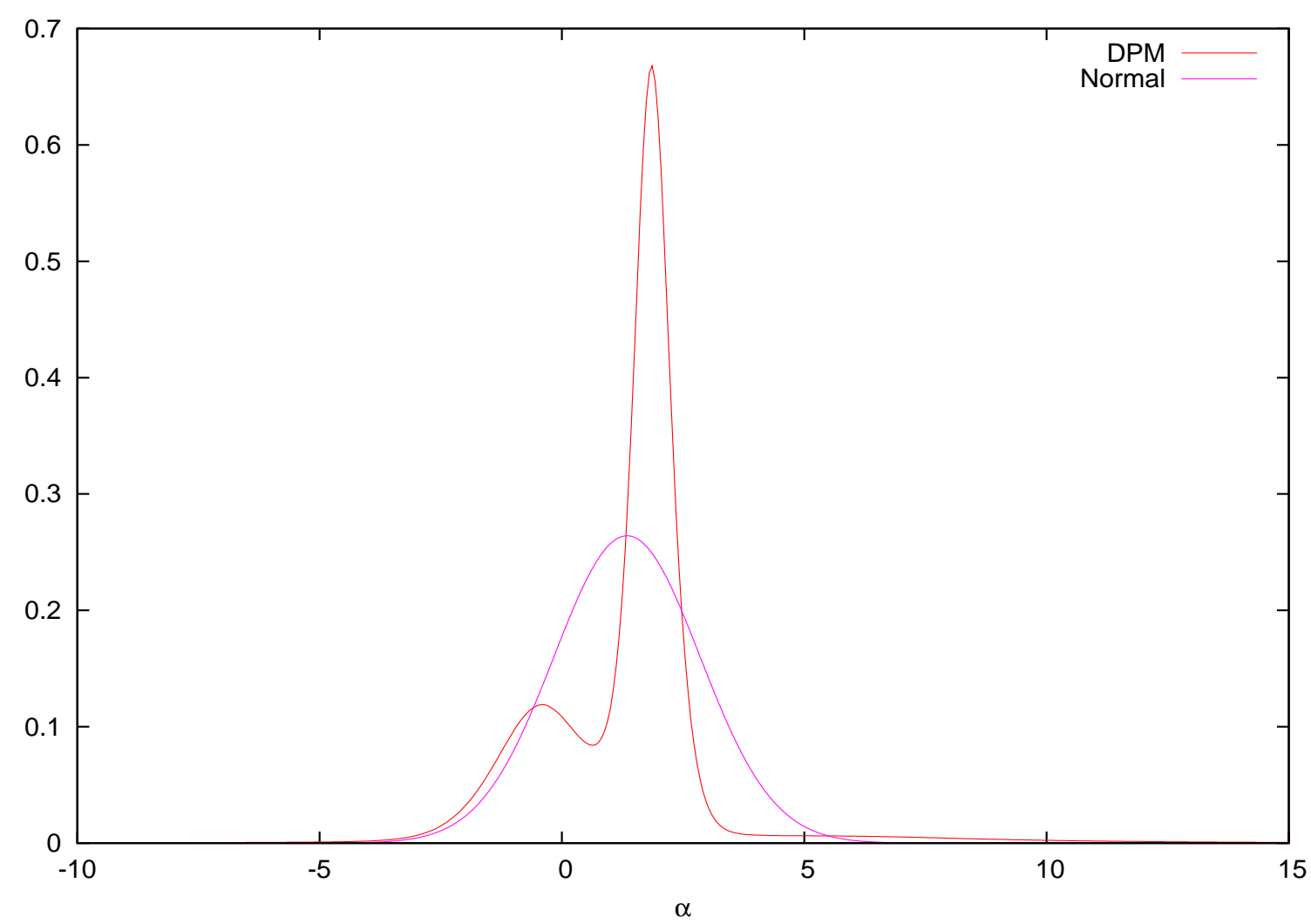

Figure 1: Posterior population distribution of alpha for the JS type investors, $\widehat{\pi}_{J S}\left(\alpha \mid r_{1}, \ldots, r_{J}\right)$, who believe the underlying population distribution is normal but with unknown mean and variance (blue line), and the posterior population distribution for our investors, $\widehat{\pi}_{D P M}\left(\alpha \mid r 1, \ldots, r_{J}\right)$, who do not assume a particular distribution for alpha but have placed a DPM prior on the unknown distribution of alpha (red line). 


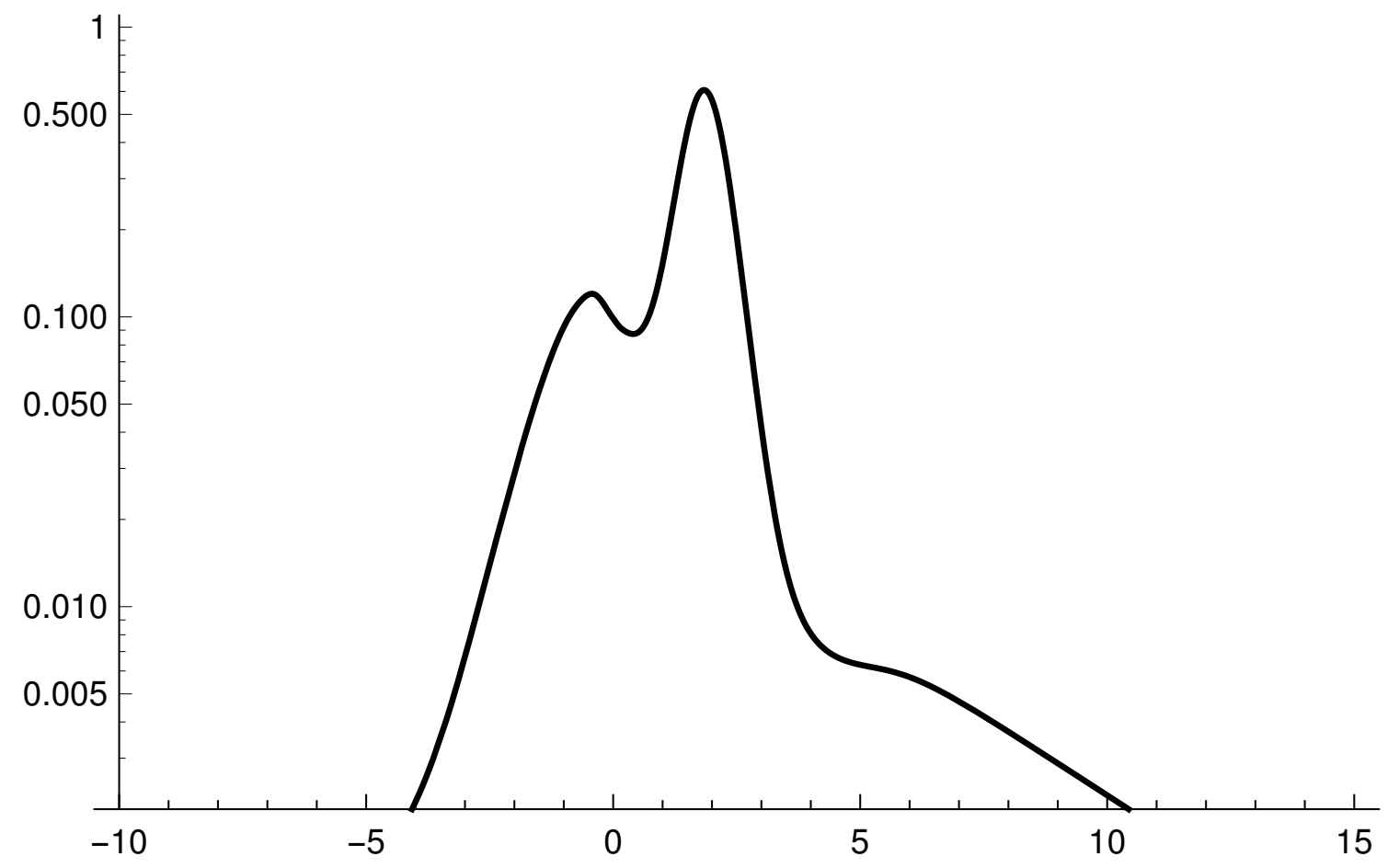

Figure 2: Log transformation of the posterior population distribution of alpha for the Bayesian nonparametric investors, $\ln \widehat{\pi}_{D P M}\left(\alpha \mid r_{1}, \ldots, r_{J}\right)$. 
In Table 1, we list the percentiles, standard deviation, skewness, and kurtosis of the parametric, and nonparametric, posterior cross-sectional distributions. Both distributions' medians are again close to the average fee of $1.5 \%$ a year. At first glance, these medians support the theoretical findings of Berk \& Green (2004) where, in the long run, successful funds break-even with an alpha that matches their fees. However, we have uncovered multiple modes. One possible explanation for these multiple modes is that funds whose alpha is close to the mode at $6 \%$ are newer funds with fewer assets under management and have not yet experienced the diminishing returns to scale assumed in the model of Berk \& Green (2004). As these young skilled funds attract assets, grow, and mature, we expect that their alphas move towards the population median; i.e., towards the break-even alpha.

\begin{tabular}{|c|c|c|c|c|c|c|c|c|c|c|}
\hline & \multicolumn{7}{|c|}{ Percentiles } & \multirow[b]{2}{*}{$\mathrm{SD}$} & \multirow[b]{2}{*}{ Skew } & \multirow[b]{2}{*}{ Kurtosis } \\
\hline & 0.01 & 0.05 & 0.1 & 0.5 & 0.9 & 0.95 & 0.99 & & & \\
\hline DP & -2.90 & -1.40 & -0.83 & 1.43 & 2.46 & 3.19 & 11.48 & 2.36 & 4.31 & 101.49 \\
\hline JS & -2.19 & -1.15 & -0.60 & 1.34 & 3.28 & 3.83 & 4.87 & 1.51 & -0.0002 & 3.02 \\
\hline
\end{tabular}

Table 1: Posterior cross-sectional percentiles, standard deviation (SD), skewness, and kurtosis for when the underlying distribution of skill is believed to be normally distributed (JS) and the nonparametric, hierarchical, prior (DPM).

According to the percentiles in Table 1, the probability of a new, or unknown fund, being extraordinarily skilled is higher than previously thought since the nonparametric population distribution's 99th-percentile is $11.48 \%$, and the 99th-percentile for the parametric distribution is only $4.87 \%$. Our flexible, nonparametric, population distribution is also more fat-tailed, with a kurtosis of 101.49, and more skewed towards finding skill in the industry with a skewness of 4.31, than the parametric population distribution. Our nonparametric population distribution also has a slightly more negative $1 \%$-percentile of $-2.90 \%$ compared to $-2.19 \%$ for the parametric distribution. So, compared to the parametric population distribution, our nonparametric approach finds that there is a greater chance a fund will be extraordinarily skilled or extraordinarily unskilled.

\subsection{Robustness to the base distribution}

To test the robustness of our nonparametric approach to the choice of $G_{0}$, or, in other words, to the prior predictive, $\widehat{\pi}_{N I G}(\alpha)$, we estimate the population distribution using a larger scale parameter, $\nu_{0}$, for the Normal, Inverse-Gamma base distribution. Since $\nu_{0}$ is the degrees of freedom of the prior predictive, increasing $\nu_{0}$ leads to a less uninformed prior predictive distribution. For $\nu_{0} \leq 0.6$ the posterior cross-sectional distributions of alpha are 
no different from the nonparametric population distribution plotted in Figure 1. However, when $\nu_{0} \geq 0.7$ the posterior population distributions are no longer multi-modal, instead, they are uni-modal with a mode near $1.4 \%$. Hence, the nonparametric distribution has fewer clusters as the degrees of freedom of its prior predictive distribution increases.

When $\nu_{0}=0.7$ the skewness of the nonparametric population distribution increased to 5.02 from the original 4.31. So when the secondary mode at the large value of alpha is not identified, we find skill to be more probable. We also find the population distribution is more fat-tailed than before with a kurtosis of 110. Hence, under the Bayesian nonparametric estimator of the population distribution, there is a greater chance a fund, for which we have no information about, being highly skilled, regardless of the value of $\nu_{0}$.

We can use the inter-quartile range of the prior predictive distribution to help explain how the choice of $\nu_{0}$ affects the number of modes of the population. The inter-quartile range for $\widehat{\pi}_{N I G}(\alpha)$ goes from a very diffuse, $10^{126}$, when $\nu_{0}=0.01$, to a relatively tight 0.18 when $\nu_{0}=0.6$. The tighter range of the prior predictive limits us from learning about the different groups of fund skill. Instead, a wider spectrum of stock picking ability gets blurred together into larger groups.

An alternative class to the Normal, Inverse-Gamma, base distribution is the flexible, but non-conjugate, Normal-SM, base distribution

$$
G_{0}\left(\mu_{\alpha}, \sigma_{\alpha}\right) \equiv N\left(\mu_{\alpha} \mid 0, s_{\mu}^{2}\right) \operatorname{SM}\left(\sigma_{\alpha} \mid 1 / 2,2, A / \sqrt{3}\right),
$$

where $\operatorname{SM}\left(\sigma_{\alpha} \mid 1 / 2,2, A / \sqrt{3}\right)$ is the base distribution for the standard deviations of the mixture.

The SM distribution is defined in Singh \& Maddala (1976) and has the density function

$$
f_{S M}\left(\sigma_{\alpha} \mid 1 / 2,2, A / \sqrt{3}\right)=\frac{3 A \sigma_{\alpha}}{\left(A^{2}+3 \sigma_{\alpha}^{2}\right)^{3 / 2}} .
$$

The SM distribution is appealing since it allows for more weight than the Inverse-Gamma distribution does for $\sigma_{\alpha}$ s close to zero. The independence between $\mu_{\alpha}$ and $\sigma_{\alpha}$ in Eq. (36) also proves advantageous since it allows the mixture location to move separately from the mixture scales. We set $s_{\mu}^{2}=2$ and $A=25$ and, because the SM is not conjugate, apply Algorithm 8 of Neal (2000) to make the draws in Step 3 of our sampler.

Under the Normal-SM, base distribution we again find the population distribution of skill to be multi-modal with modes located near those of the nonparametric density in Figure 1. ${ }^{19}$ The posterior draws of $K$ range from four to fourteen clusters with a median of six

\footnotetext{
${ }^{19}$ The posterior results using the Normal-SM distribution are available upon request.
} 
clusters. Hence, the number of mixture clusters is marginally larger under the normal-SM base distribution than the Normal, Inverse-Gamma.

The posterior mean of the concentration parameter, $B$, is also slightly larger under the Normal-SM base at 0.59 compared the Normal, Inverse-Gamma's 0.125. Both of these posterior estimates of $B$, along with $K$, support our earlier conclusion that the population distribution of skill is neither normally distributed, nor is skill idiosyncratic to a fund. Instead, the population distribution of skill requires the flexibility that our Bayesian nonparametric approach provides.

\subsection{Evolution of the population}

Beginning in 1993 the number of new mutual funds entering the actively managed fund industry accelerated. Following 1993 more than three-hundred mutual funds opened each year. Entry peaked in 1998 with 659 funds opening up for business. This history of funds opening for business allows us to analyze how the population of skill evolved over this time period and also investigate if these new funds were more skilled than the old ones.

Starting in 1981 we move forward in one-year increments up to the year 2000 and estimate the cross-sectional distribution of alpha using the return histories of all the funds to have ever existed up to the specified year. ${ }^{20}$ We find that there are four episodes or eras for the population distribution of skill; i) 1981 to 1897, ii) 1988 to 1993, iii) 1994 to 1996, and iv) 1997 to 2000. In Figure 3 we plot the population distributions from each of these eras in the figure's four panels.

In the first panel of Figure 3, we plot the seven posterior cross-sectional distributions from the growing number of fund histories beginning in 1981 and ending in 1987. Each distribution is symmetrical around the average fee of $1.5 \%$. This symmetry indicates funds are skilled enough on average to cover their costs and equally likely to cover or not cover their fees.

As the entry into the mutual fund industry accelerated during the 1988 to 1993 period, the population distribution in the second panel of Figure 3 tightens around the mean. During this era, the probability of a fund selecting stocks that will result in an abnormally negative alpha declines relative to the earlier episode as the left-hand tails for these distributions are now thinner. Probability of finding highly skilled funds are also on the increase as the right-hand tail of the population pushes out past six percent to eight percent. Hence, the entry of new funds during this era and the performance of existing funds improved the overall performance of the population.

\footnotetext{
${ }^{20}$ New mutual funds were included when they had 4-months worth of returns.
} 

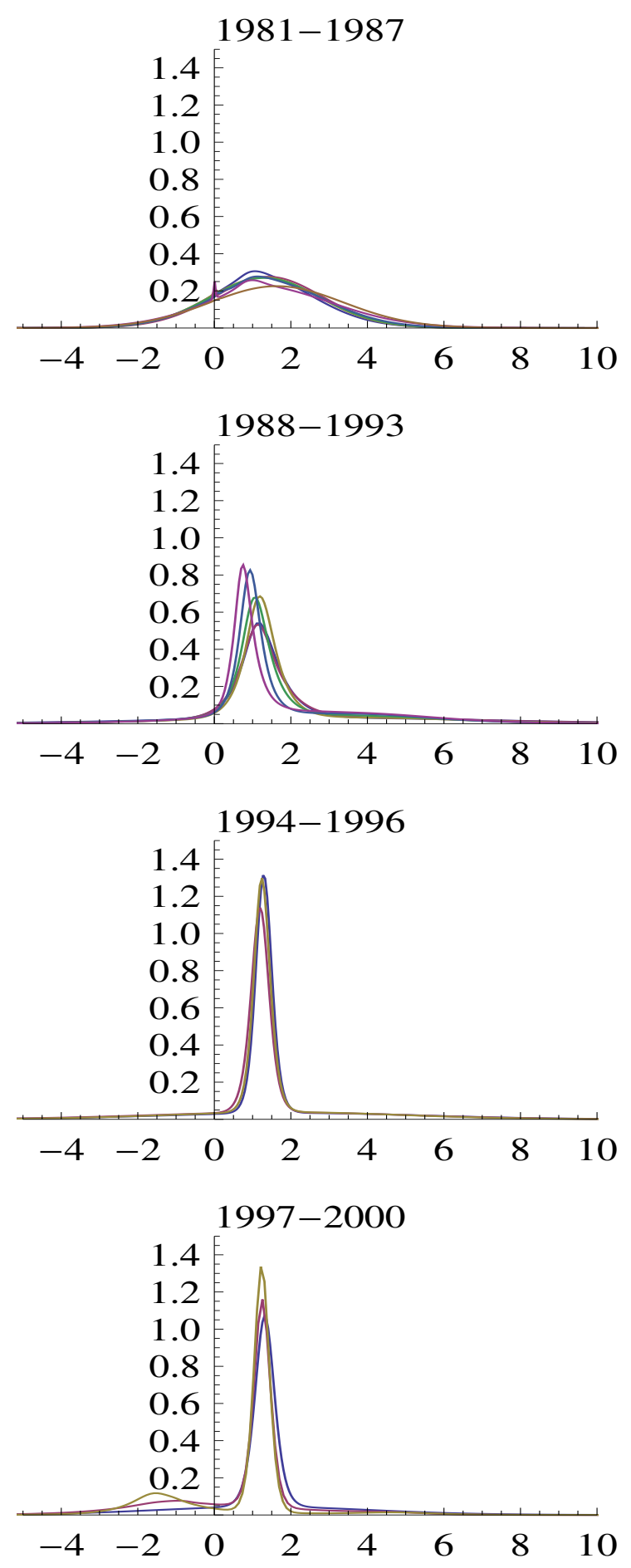

Figure 3: Posterior cross-sectional distributions of alpha starting with the return histories of funds up to 1981 and then incrementing forward one year at a time from 1982 to 2000. More formally, plots of $\widehat{\pi}_{D P M}\left(\alpha \mid R_{t}\right), t=1981, \ldots, 2000$, where $R_{t}$ are the return histories of all the mutual funds ever in business during the 1961 to year $t$ time period. A new fund is only included if it has a history of least four months. Each panel contains the population distributions from that era; i) 1981-1987, ii) 1988-1993, iii) 1994-1996, iv) 1997-2000. 


\begin{tabular}{rrrrrrrc}
\hline Year & $J_{t}$ & $K_{t}$ & Median & Skew & $p_{0.05}$ & $p_{0.95}$ & $P(\alpha>0)$ \\
\hline \hline 1981 & 328 & 1 & 1.220 & 0.068 & -1.139 & 3.700 & 0.812 \\
1982 & 348 & 1 & 1.314 & 0.262 & -0.957 & 4.069 & 0.853 \\
1983 & 382 & 1 & 1.296 & 0.042 & -1.121 & 3.774 & 0.816 \\
1984 & 432 & 1 & 1.318 & 0.065 & -1.122 & 3.888 & 0.820 \\
1985 & 487 & 1 & 1.395 & 0.011 & -1.173 & 3.982 & 0.816 \\
1986 & 577 & 2 & 1.234 & 0.379 & -1.342 & 4.987 & 0.838 \\
1987 & 665 & 1 & 1.595 & 0.078 & -1.288 & 4.654 & 0.828 \\
1988 & 778 & 2 & 1.305 & 1.041 & -1.795 & 7.516 & 0.892 \\
1989 & 854 & 2 & 1.329 & 1.016 & -1.368 & 7.703 & 0.900 \\
1990 & 902 & 2 & 1.279 & 0.571 & -0.951 & 6.203 & 0.921 \\
1991 & 983 & 2 & 1.185 & 1.124 & -0.782 & 6.015 & 0.920 \\
1992 & 1073 & 3 & 1.036 & -0.521 & -1.096 & 5.662 & 0.914 \\
1993 & 1258 & 3 & 0.963 & -0.477 & -0.846 & 5.970 & 0.918 \\
1994 & 1599 & 2 & 1.303 & 0.861 & -1.151 & 5.373 & 0.922 \\
1995 & 1939 & 2 & 1.210 & 0.544 & -1.711 & 5.194 & 0.902 \\
1996 & 2275 & 2 & 1.270 & 0.608 & -1.183 & 4.890 & 0.918 \\
1997 & 2704 & 2 & 1.314 & -0.064 & -1.540 & 4.150 & 0.900 \\
1998 & 3364 & 3 & 1.164 & -4.182 & -2.695 & 3.065 & 0.803 \\
1999 & 3977 & 4 & 1.160 & -1.448 & -2.188 & 2.119 & 0.778 \\
2000 & 4539 & 3 & 1.444 & 4.584 & -0.766 & 4.719 & 0.927 \\
\hline
\end{tabular}

Table 2: Yearly evolution of the median, skewness, and probability of beating the passive four-factor portfolio, $P(\alpha>0)$, where $J_{t}$ is the number of mutual fund, both alive and dead, at year $t, K_{t}$ is the posterior median number of clusters, and $p_{0.05}$, and $p_{0.95}$, are the 5 th and 95th percentiles of the cross-sectional mutual fund performance distribution. 
During the 1994 to 1996 era, the population distributions continue to tighten around the mean. However, after 1997 the population begins to change. In the bottom panel of Figure 3, the population distributions start to skew to the left. Ultimately a second mode appears at a negative alpha. This last era corresponds to the fastest growing period of the mutual fund industry and, according to the population distributions, poorer stock-picking ability.

In Table 2 we list the characteristics and features of the cross-sectional distributions from Figure 3. Each line contains the total number of funds, both in, and out of business, since 1961 up to that year, $J_{t}$, the posterior median of the number of clusters, $K_{t}$, the median and skewness, and the 5th-percentile, $p_{0.05}$, and the 95th-percentile, $p_{0.95}$, of the cross-sectional distribution, and the probability of an unknown mutual fund generating a positive alpha, $P(\alpha>0)$. Beginning in the 90s any arbitrary fund is exceptionally skilled, as defined by having an alpha in the top $5 \%$ of the distribution if it generated an alpha of two to six percent. This value of alpha is smaller than a highly skilled fund from the $80 \mathrm{~s}$. For example, over the 90 s the 95 th-percentile declined from approximately $6 \%$ to $2 \%$ per annum. In contrast these percentiles were never less than $3.7 \%$ in the $80 \mathrm{~s}$ and reached a high of $7.7 \%$ in 1989.

During the 90s the alpha of an unskilled fund, as defined by the 5th-percentile, also declined but in a more noisy fashion than did the alpha for a skilled fund. Poor performance in the mutual fund industry went from $-0.95 \%$ in 1990 to a low of $-2.7 \%$ in 1998 . Except for 1998 and 1999, the probability of an unknown mutual fund being capable of generating positive alphas stayed right around $90 \%$. Thus, overall mutual fund performance went down during the 90 s relative to the $80 \mathrm{~s}$.

In Figure 4 we plot the population distributions of alpha from 1995 to 2001 using only the return histories of those funds that opened for business during the 1993 to 2001 period. ${ }^{21}$ Funds that were new to the industry were more likely to generate a positive alpha as seen in the positive primary mode. However, over this same period the probability of a new fund generating a negative alpha is also increasing as the negative mode moves further to the left. Thus, we conclude that during the latter half of the 90s when the number of new funds entering into mutual fund industry was accelerating, a new fund was likely to be skilled and capable of covering its fees, but with each year there was an increasing chance the new fund would be unable to earn a high enough return to justify its fees.

\footnotetext{
${ }^{21} \mathrm{~A}$ new fund was only included if it had twelve months of return performance.
} 


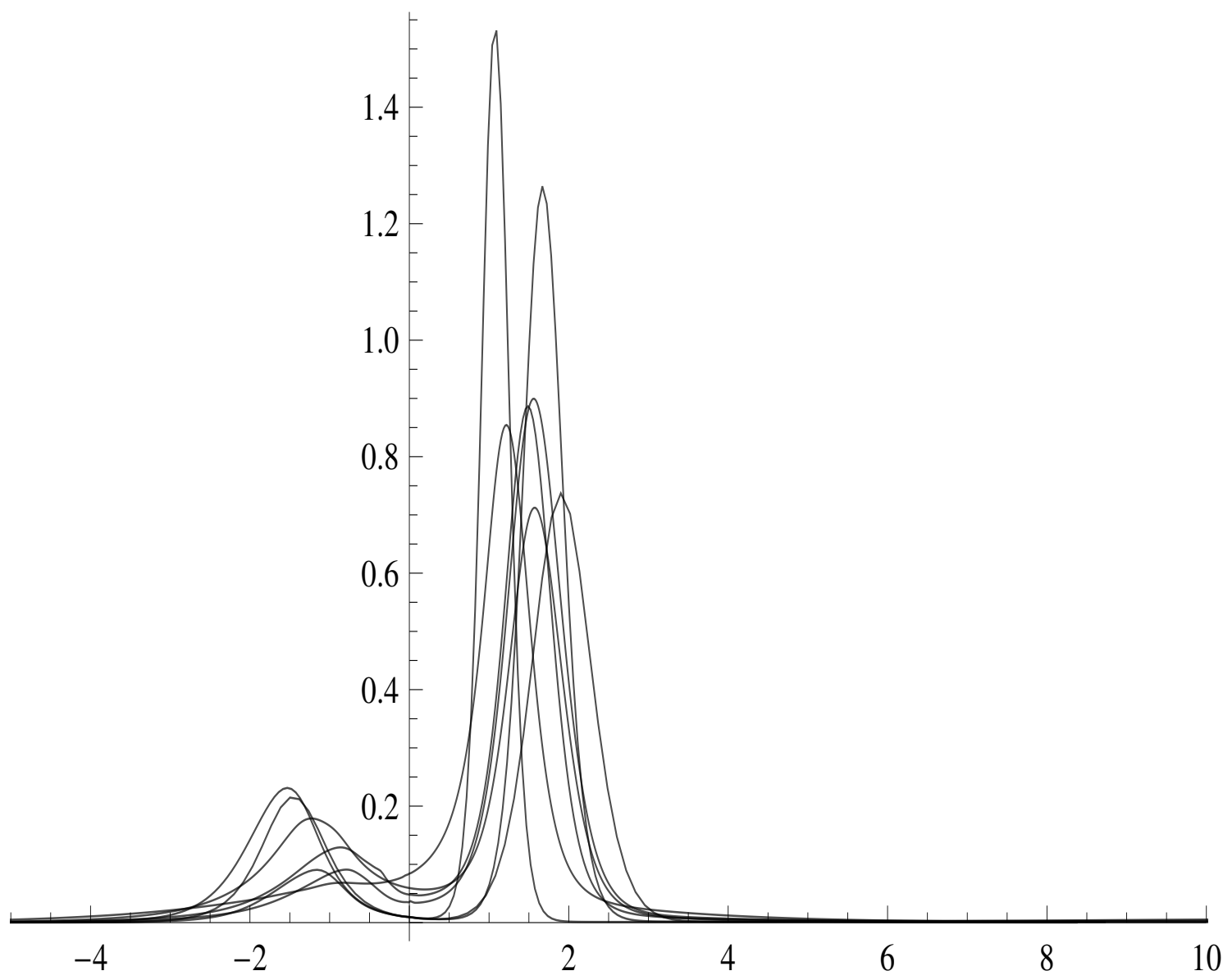

Figure 4: Posterior cross-sectional distribution of alpha from 1995 to 2001 using only the performance histories of the funds that entered the business after 1992 and had a years worth of performance data. 


\subsection{Comparison of the individual alphas}

In Figure 5 we plot each of the 5,136 mutual fund's 95\% HPD interval for its alpha along with the posterior median (represented as dots when visible). At the top of the figure are the posterior results for the funds with the shortest histories, and at the bottom are the results for the ones with the longest.

We calculate the 95\% HPD intervals using three different approaches. Panel (a) of Figure 5 plots the HPD intervals where ability is believed to be idiosyncratic to the mutual fund and the prior for alpha is $N\left(0, s_{0}^{2}\right)$, with $1 / s_{0}^{2}=0 .{ }^{22}$ Panel (b) plots the HPD intervals using the approach of JS. The population is assumed to be normally distributed but the mean and variance are unknown and modeled with the uninformative Jeffreys prior, $\pi\left(\mu_{\alpha}, \sigma_{\alpha}^{2}\right) \propto 1 / \sigma_{\alpha}^{2}$. Panel (c) of Figure 5 plots the 95\% HPD interval of each fund's alpha using our Bayesian nonparametric approach. Our initial guess for the cross-sectional distribution of alpha is again the diffuse Student-t distribution found in Eq. (19) whose mean is zero, scale 0.0011, and with 0.1 degrees of freedom.

\footnotetext{
${ }^{22}$ Because this is the Jeffreys prior the intervals in Figure 5 Panel (a) are equivalent to the 95\% confidence intervals of the ordinary least squares estimate of alpha.
} 


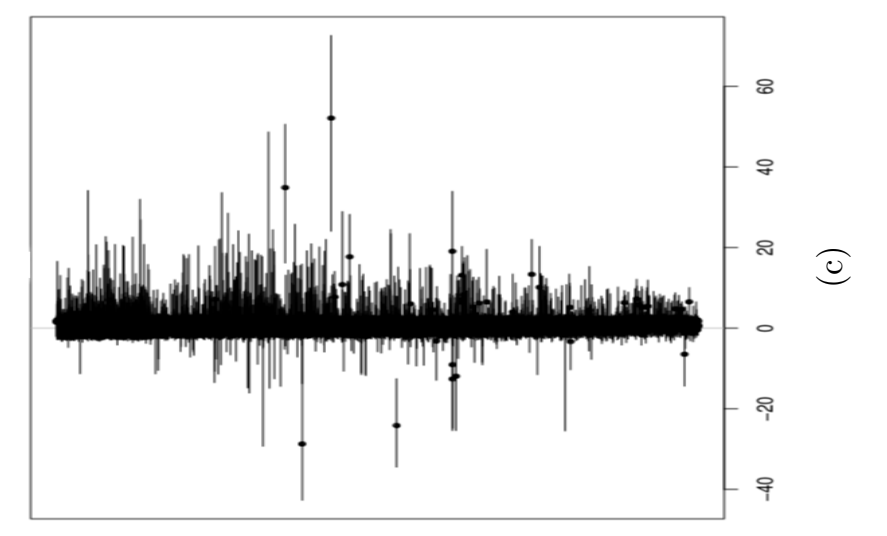

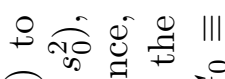

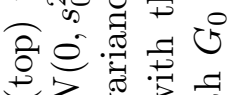

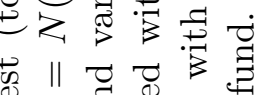

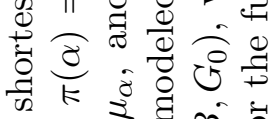

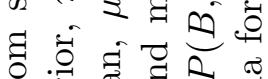

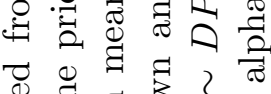

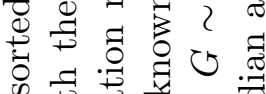

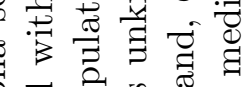

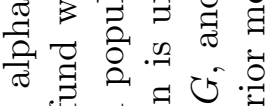

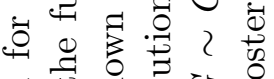

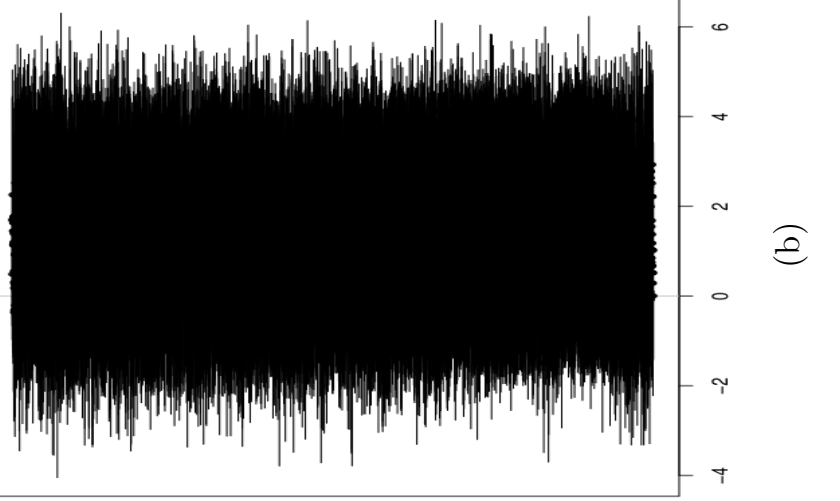

సึ

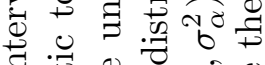

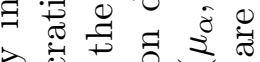

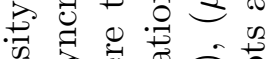

过

ᄀ.

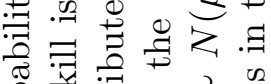

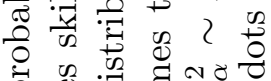

है

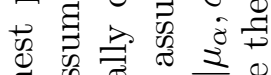

兽

- $\approx \overrightarrow{0}$

ถู

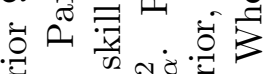

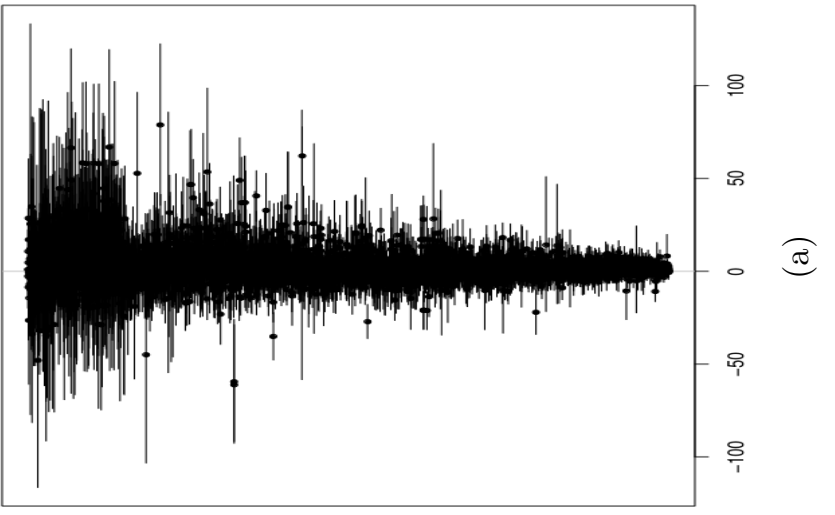

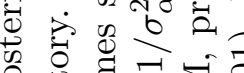

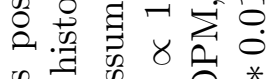

है चี तิ

$\exists \exists_{0}^{0} 0$ b

ग्ञ

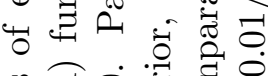

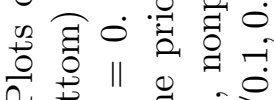

A

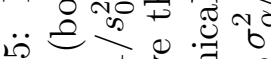

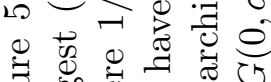
焉 
Comparing the funds' posterior HPD intervals plotted in the three panels of Figure 5, it is clear that what one assumes about the nature of the population distribution affects a particular mutual fund's estimated alpha. The posteriors in Panels (b) and (c) draw on the performance of other funds to make an informed guess about the stock-picking ability of a particular fund. By borrowing information from other funds, the HPD intervals in these two panels are tighter than those in Panel (a). As a result, the posteriors in Panel (b) and (c) use information from the population to be more precise about a fund's future ability to produce excess returns than the idiosyncratic approach used in Panel (a).

Because the posteriors in Panel (a) view skill idiosyncratically the length of a fund's performance window influences the posteriors. Short-lived funds found at the top of Figure 5(a) have larger and noisier HPD intervals than do the long-lived funds located at the bottom. Although there are also funds with long performance histories that have wide HPD intervals due noisy and erratic performance histories.

At the other end of the spectrum are the tight and homogeneous HPD intervals in Panel (b) of Figure 5. Believing a fund's performance comes from a normal, cross-sectional, distribution with an unknown population mean and variance shrinks a fund's estimated alpha towards the average alpha of the industry. Exceptionally skilled funds get pooled together with average funds, and funds with short or noisy performance histories take on the skill characteristics of the population. Hence, the homogeneity of the HPD intervals in Panel (b).

By treating all the alphas as draws from a normal population distribution, unskilled funds, like the one near the top of Figure 5 (a) where the median alpha is close to $-50 \%$, look better in Panel (b) than maybe they should. Furthermore, a highly skilled fund like those in Panel (a) with posterior medians greater than $50 \%$ do not look so extraordinary in Panel (b). Our Bayesian nonparametric learning approach automatically determines if such funds should be pooled together or treated separately. In contrast to Panel (b), where $K$ is set equal to one, a priori, the intervals calculated with our nonparametric approach in Panel (c) randomly group together similarly skilled funds and integrate away the uncertainty of $K$. Conditional on the random group, information is borrowed from the other funds in the group and used to infer each of the funds alphas.

The benefits from letting $K$ be unknown is found in the alpha for the Schroder Ultra Fund. In Figure 5 (c), Schroder Ultra has the highest posterior median alpha of all the funds at $50 \%$ per annum. The next closest fund is the Turner Funds Micro Cap Growth fund at $33 \%$. Our nonparametric sampler in Section 5 randomly groups Schroder Ultra with other funds. Given the likely small size of this random, but highly skilled, group, the variance 
of the group, $\sigma_{\alpha, k}^{2 *}$, is likely large. According to Eq. (28), this large variance causes Step 2 of the sampler to make posterior draws of the Schroder fund's alpha that on average are weighted more towards the sample average of the risk-factor adjusted returns of the fund,

$\mathcal{T}_{i}^{-1} \sum_{t=\tau_{i}}^{T_{i}} r_{i, t}^{*}$, and weighted less toward the average of the group, $\mu_{\alpha, k}^{*}$. In the extreme case where a fund has no peers our Bayesian nonparametric approach essentially treats the fund idiosyncratically as in Panel (a). As a result, the Schroder Ultra fund's HPD intervals and medians in Panels (a) and (c) are very similar. This similarity stands in stark contrast to Panel (b) where, because of the shrinkage towards the population average, the posterior alpha does not even put Schroder Ultra among the top ten performing funds.

\subsection{Shrinkage}

To determine how much a specific mutual fund's alpha is affected by one's beliefs about the cross-sectional distribution of mutual fund performance in Figure 6 we graph two scatterplots. In each scatter-plot we plot on the $y$-axis the posterior mean alpha for each of the 5,136 funds using our Bayesian nonparametric method. Panel (a) plots these nonparametric posterior mean alphas against the posterior mean alpha where skill is believed to be idiosyncratic. In Panel (b) we graph on the $x$-axis the posterior mean of the alphas where skill is believed to be normally distributed. The forty-five degree line in both plots shows where the assumption about the cross-sectional distribution does not affect the estimate of a fund's alpha relative to our nonparametric approach.

In Panel (a) of Figure 6 every mutual fund's expected level of skill has moved, to varying degrees, away from the posterior beliefs of someone who believes the skill of funds is idiosyncratic and towards zero; i.e., the points have moved vertically away from forty-five degree line towards zero. Hence, those who believe there is an unknown cross-sectional distribution of skill underlying each funds performance level, and learns about it, discovers that funds identified by those who view skill idiosyncratically as being skilled (unskilled) are less (more) capable of selecting stocks that beat the market.

There are a handful of points in Figure 6(a) where skill is so unique to the fund that treating them idiosyncratically is only slightly different from our nonparametric estimates. These funds are those whose posterior mean alpha are closest to the forty-five degree line in Panel (a) and include both skilled and unskilled funds. In general, there are fewer extraordinary funds when we do not treat skill idiosyncratically, but instead treat each fund's performance as a draw from an unknown population distribution. By flexibly learning how skill is distributed over the mutual fund industry we identify actual fund-specific performance skills while guarding against the noisy performance measures the idiosyncratic 


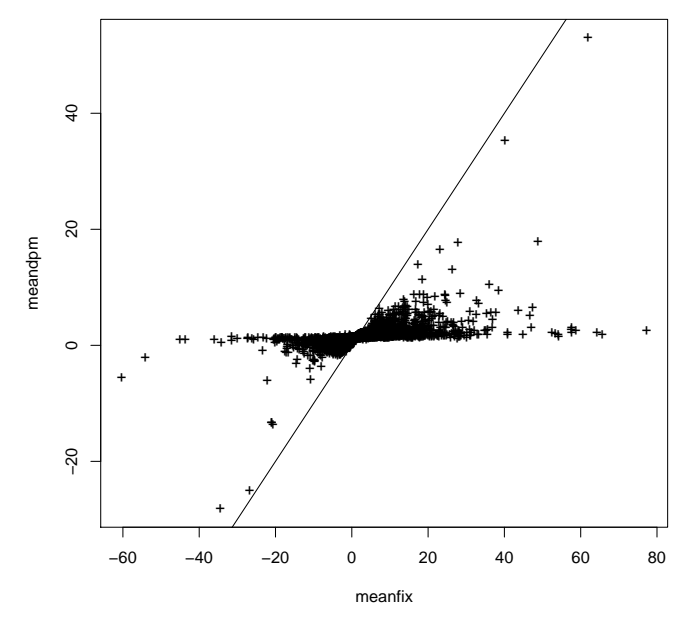

(a)

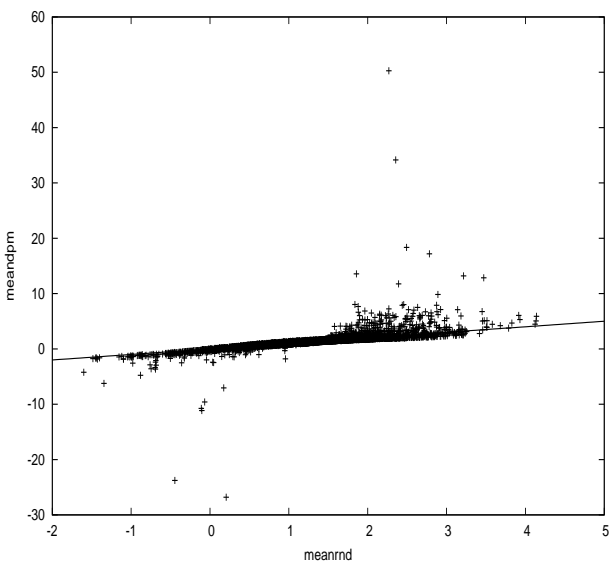

(b)

Figure 6: Scatter plots of the 5,136 fund's posterior mean alphas where in both Panel (a) and Panel (b) the y-axis are the posterior mean under our nonparametric hierarchical prior. The $\mathrm{x}$-axis of Panel (a) are the posterior mean alphas when funds are treated idiosyncratically and the alphas' priors is the Jefferys prior. In Panel (b) the x-axis are the posterior mean alphas under a normal prior whose mean and variance are unknown and modeled with the Jefferys prior, $\pi\left(\mu_{\alpha}, \sigma_{\alpha}^{2}\right) \propto 1 / \sigma_{\alpha}^{2}$. To provide a point of reference a 45 -degree line has been added to both panels to represent those posterior means where the two relative approaches have the same guess for alpha. 
approach finds for short lived funds.

Figure 6(a) also helps us answer the question posed by Kosowski et al. (2006) and Fama \& French (2010) as to whether skilled funds are genuinely talented or just lucky. We find luck playing little to no role in the success of the funds whose posterior mean for alpha is greater than $4 \%$ on both the vertical and horizontal axis. Because the posterior crosssectional distribution of skill is so informative about the typical fund's ability, posterior knowledge about the skill level of an exceptional fund is distinctly different from the posterior population distribution. In Figure 6(a), these exceptionally skilled funds were not lucky but were truly skilled since many of their posterior means are greater than $4 \%$ on both the vertical (nonparametric hierarchical prior) and horizontal (idiosyncratic priors) axis. A lucky fund is one of the many "skilled" funds whose posterior mean on the horizontal axis is larger than $5 \%$, for instance, the extreme fund whose posterior mean is nearly $80 \%$, but then shrinks close to zero under the nonparametric hierarchical prior. Later in Table 3 we list the names of these highly skilled funds.

In contrast to Panel (a), many of the points in Panel (b) of Figure 6 lie on the forty-five degree line. These alphas belong to funds having the same average ability and variance as that of the normal cross-sectional distribution. Modeling the performance of this particular group of funds as draws from a normally distributed cross-sectional would not interfere with the expected value of the posterior alphas. However, identifying this group of funds from the cross-section of funds, a priori, would be impossible but is something our nonparametric approach does naturally as it learns about the population distribution.

From the off-diagonal points in Panel (b) of Figure 6 we discover some funds' alpha are drawn from groups whose means and variances are different from the global average level of skill and its variance. If the population is assumed to be normally distributed many abnormally skilled and unskilled funds would go undetected. For example, when we learn the population distribution we find that the posterior alpha for Potomac OTC/Short fund under performs the market by an average of $27 \%$ a year instead of having an alpha greater than zero (Potomac is the worse performing fund in our panel of mutual funds whose posterior mean alpha in both panels of Figure 6 is the point located at the very bottom). In stark contrast, those who believe skill is normally distributed over the population believe the Potomac OTC/Short fund can produce on average an excess market return of less than $1 \%$ per year (located on the $\mathrm{x}$-axis of Panel (b)).

The Potomac fund is not unique in this respect. Many other extraordinary skilled and unskilled funds look quite ordinary to those who believe skill is normally distributed across funds. Clearly the assumption of a normal population distribution for mutual fund skill 
adversely affects the estimate of these funds alphas. ${ }^{23}$

\subsection{Posterior distribution of each funds' alpha}

To evaluate and compare the skill level of the funds in our panel we plot in Figure 7 the posterior distribution of alpha for all 5,136 mutual funds. The posteriors for the 3,844 mutual funds that were still open for business at the end of our sample are plotted in blue lines. The posterior distributions for the 1,292 funds that were no longer in business at the end of the sample are plotted in red. Transparency has been added to the densities in the figure so that darker shades of red, blue, or purple (combinations of red and blue) show where the posterior distributions are concentrated.

As we showed in Eq. (7), a fund's posterior is proportional to

$$
\pi\left(\alpha_{i} \mid r_{1}, \ldots, r_{J}\right) \propto \pi\left(\alpha_{i} \mid r_{-i}\right) f\left(r_{i}^{*} \mid \alpha_{i}\right), \quad i=1, \ldots, J
$$

where $r_{i}^{*}$ is the risk and factor adjusted return history of the $i$ th fund. ${ }^{24}$ In other words, when the return histories of the other $J-1$ funds, $r_{-i}$, have been observed, but before the $i$ th funds returns, $r_{i}$, are seen, our understanding of the $i$ th fund's skill level is best described by the "updated prior" distribution, $\pi\left(\alpha \mid r_{-i}\right)$; i.e., the posterior population distribution or posterior predictive distribution.

By Bayes rule how different a fund's posterior, $\pi\left(\alpha_{i} \mid r_{1}, \ldots, r_{J}\right)$, is from the posterior predictive distribution, $\pi\left(\alpha_{i} \mid r_{-i}\right)$, is how exceptional the fund is from a fund we have no information about. The more uniquely shaped a fund's posterior the more its likelihood, $f\left(r_{i}^{*} \mid \alpha_{i}\right)$, plays in our understanding of its ability. Because our panel consists of 5,136 funds the skewness, kurtosis, and multi-modality of the nonparametric posterior population distribution in Figure 1 is unaffected when we drop any one fund from the panel. Given this robustness of the posterior population distribution we define a fund as being exceptional when its posterior for alpha does not have the same multi-modal shape as $\widehat{\pi}_{D P M}\left(\alpha \mid r_{1}, \ldots, r_{J}\right)$. To identify these exceptional funds we plot in orange the posterior population distribution in Figure 7.

Many of the posterior distributions in Figure 7, be the fund dead or alive, resemble the multi-modal, cross-sectional distribution of alpha. The large number of funds whose posterior is similar to the population shows how important the posterior population distribution is in determining the skill level of a fund, and, hence, how vital it is to correctly

\footnotetext{
${ }^{23}$ The performance measure of JS and Cohen et al. (2005) both suffer from this type of shrinkage toward the average of the overall population.

${ }^{24} \mathrm{We}$ could integrate out the betas and the return variance in which case a fund's likelihood function, $f\left(r_{i} \mid \alpha_{i}\right)$, is a Student-t distribution but we would require a different sampler for the DP unknowns.
} 


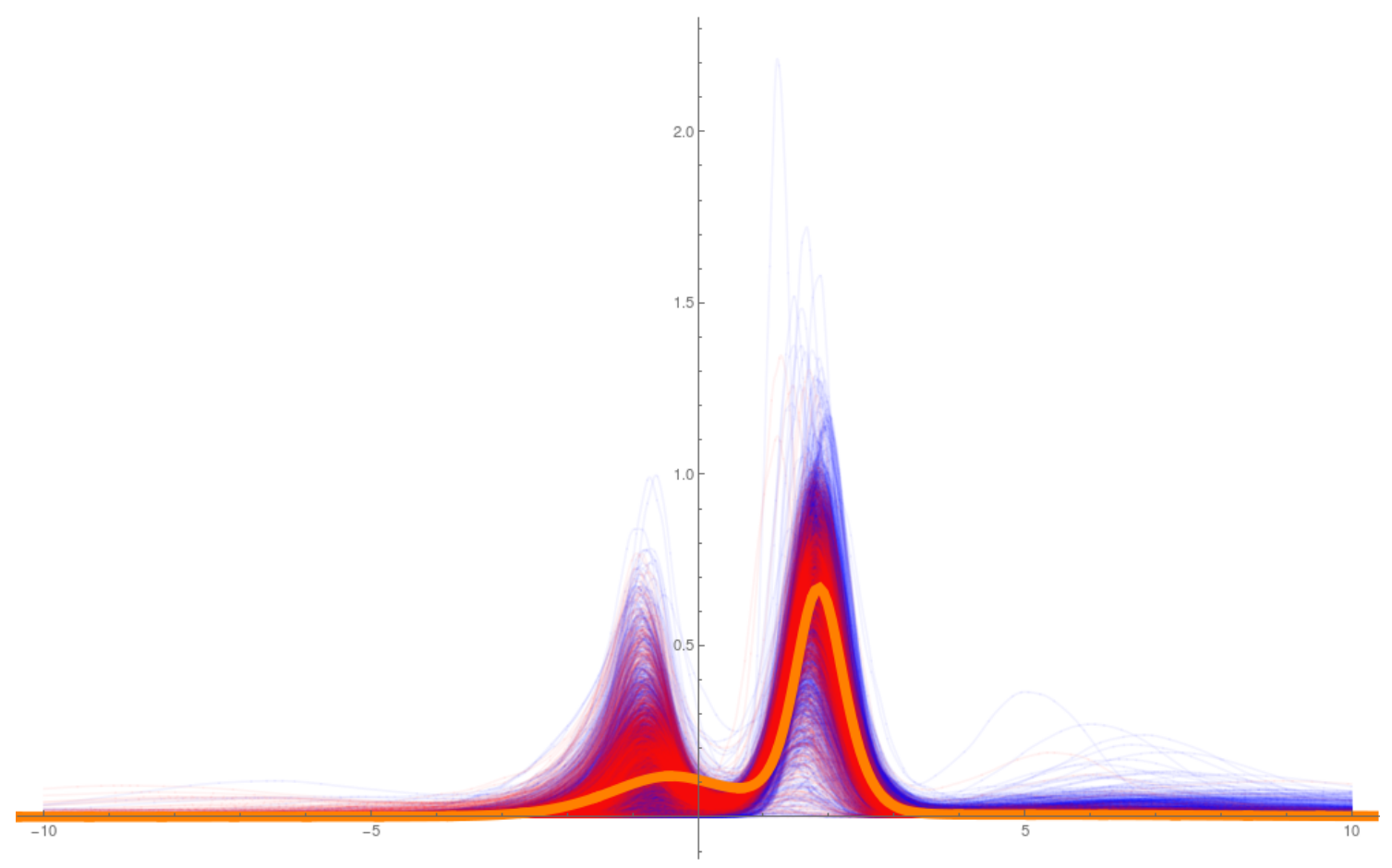

Figure 7: Posterior cross-sectional distribution of alpha in orange and every funds posterior distribution of alpha, $\pi\left(\alpha_{i} \mid r_{1}, \ldots, r_{5136}\right), i=1, \ldots, 5136$, where the funds that were still in business at the end of the sample are plotted in blue, whereas those funds that were not in business are in red. Darker shades of red or blue indicate a higher concentration of funds having similar shaped posteriors. 
model the population. It also shows how little information there is about skill in most mutual funds return history. ${ }^{25}$ For the typical fund whose performance is average and has a likelihood which is relatively flat, the posterior cross-sectional distribution resolves most of the uncertainty in alpha.

We draw three conclusions about the skill level of the funds from Figure 7 . First, regardless of a fund being in or out of business, its posterior in general has two modes. The secondary modes are close to $-1 \%$, indicating that the funds have the potential to generate losses. The primary modes are close to $2 \%$. Thus, many of the 5,136 funds are likely to cover their fees but there is a non-trivial chance the fund will fail to earn a return that covers its costs.

The second finding from the posteriors of Figure 7 is the credible evidence of there being a few exceptional funds. These funds can be exception either because they are unskilled or because they skilled. There are twenty-one exceptionally skilled where there is a $95 \%$ probability of its alpha being greater than $1.5 \%$, and fifty exceptionally unskilled that have the same probability of being less than $1.5 \%$. Finding so few exceptional funds runs counter to earlier empirical findings where there is a larger presence of skilled and/or unskilled funds (see Kosowski et al. (2006), Fama \& French (2010), and Barras et al. (2010), and Ferson \& Chen (2015)). However, as we have already pointed out these earlier findings suffer from noisy alpha estimates. Hence, we conclude that most mutual funds are not extraordinarily talented or unskilled. Instead, most funds have a greater chance of being just talented enough to select stocks that on average result in a return that justifies its costs, expenses, and fees.

Our third finding concerns those funds that are exceptionally unskilled but for some reason were still in business. A number of predictive densities in Figure 7 have fat tails over negative values of alpha and were not out of business. In Figure 8(a) we plot in blue the alive fund's posterior and in red the dead fund's posterior distributions for the fifty exceptionally unskilled funds. Twenty-nine of these poor performing funds were still in business at the end of our sample. This includes the worse performing fund from our panel - the Potomac OTC/Short fund, whose expected alpha is $-26 \%$ and has a $3 \%$ chance of losing between $20 \%$ to $40 \%$ percent a year.

Except for the two closed funds, Bowser Growth Fund, whose expected alpha is $-24 \%$, and Ameritor Industry Fund, whose expected alpha is $-7 \%$, the other forty-seven unskilled funds engage in trades that are expected to result in gross losses of between zero to five

\footnotetext{
${ }^{25}$ Not being able to accurately estimate a funds alpha with only its return history has been a well known problem with ordinary least square estimates of the alpha. The mutual fund skill literature is full of results where the $R^{2}$ s is small.
} 


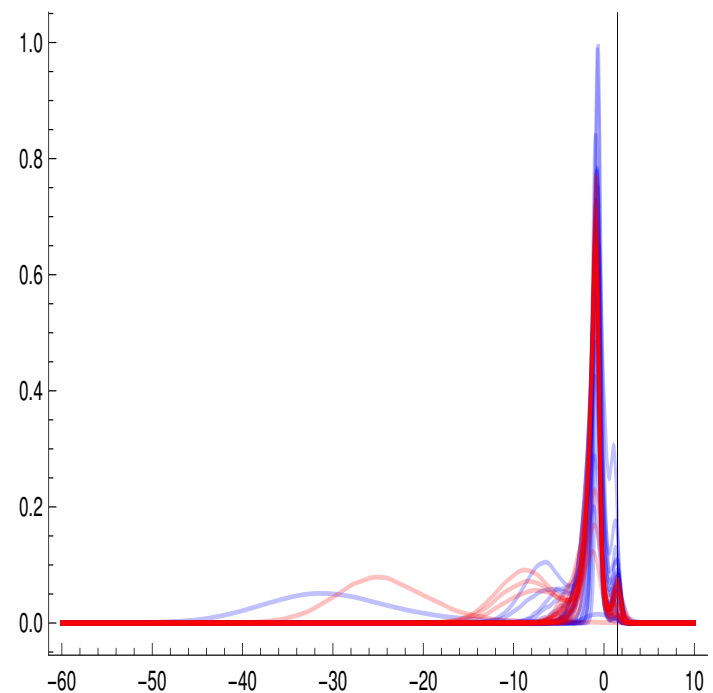

(a) Unskilled funds

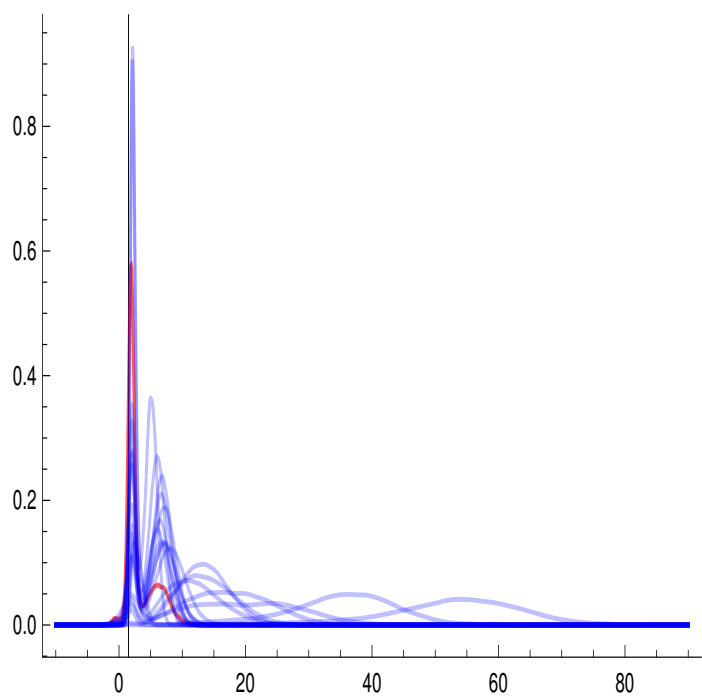

(b) Skilled funds

Figure 8: The posterior distribution of alpha where Panel (a) plots the posteriors of the fifty unskilled fund's who have at least a $95 \%$ chance its alpha will be less than 1.5 percent, and Panel (b) are the posteriors for the twenty-one skilled funds who have at least a 95\% chance its alpha will be greater than 1.5 percent (the vertical line in both plots is at the average fund's fee of 1.5 percent). Posteriors for the funds that are in business at the end of the sample are plotted in blue, whereas those that have closed are in red. In Panel (a) there are a total of twenty-one dead unskilled funds. In Panel (b) there is only one dead skilled fund (T. Rowe Price Capital Appreciation Fund).

percent a year. Nine of these funds had been in business since the early 1960s and were still in business in 2001. Thus, we conclude that poor performance by a fund does not necessarily lead investors to divest their money and cause such funds to go out of business. Perhaps there are restrictions in place where investors are prohibited from withdrawing their investment or redemptions are limited.

In Figure 8(b) we plot the posterior distribution of the twenty-one exceptionally skilled funds. Notice how different these funds' posteriors are from the nonparametric posterior population distribution of Figure 1. These funds have densities where the weight assigned to the alphas between $5 \%$ to $10 \%$ are noticeably greater than the population distribution. A few of these exceptionally skilled funds have a primary mode located at an alpha larger than the primary mode of the population. Half of these funds have a primary mode near $1.5 \%$. Only one of the funds was no longer in business.

In Table 3 we list from shortest to longest return histories the twenty-one skilled funds posterior mean of alpha and the number of years the fund had been in business. Four of 


\begin{tabular}{lcc} 
Fund & Years of Operation & Alpha \\
\hline \hline Turner Funds: Micro Cap Growth & $1998-2001$ & 33.07 \\
Schroeder Ultra Fund & $1997-2001$ & 49.77 \\
Artisan Mid Cap Fund & $1997-2001$ & 16.77 \\
Needham Growth Fund & $1996-2001$ & 17.40 \\
Olstein Financial Alert Fund/C & $1996-2001$ & 13.84 \\
Fremont Mutual Fds:US Micro Cap Fund & $1994-2001$ & 13.23 \\
PIMCO Funds:Stocks Plus Fund/Instl & $1993-2001$ & 2.46 \\
Fidelity Dividend Growth & $1993-2001$ & 4.87 \\
Managers Funds:US Stock Market Plus & $1992-2001$ & 2.44 \\
Fidelity Low Priced Stock & $1990-2001$ & 5.93 \\
Victory Funds:Diversified Stock Fund/A & $1989-2001$ & 2.88 \\
T Rowe Price Capital Appreciation Fund & $1986-1999$ & 3.75 \\
JP Morgan Growth \& Income Fund/A & $1987-2001$ & 6.30 \\
Gabelli Growth Fund & $1987-2001$ & 5.29 \\
Weitz Series Fund:Value Portfolio & $1986-2001$ & 4.50 \\
IDEX Janus Growth Fund/A & $1986-2001$ & 4.87 \\
Gabelli Asset Fund & $1986-2001$ & 4.99 \\
Oppenheimer Growth/A & $1973-2001$ & 3.82 \\
AXP Growth Fund/A & $1972-2001$ & 4.71 \\
Janus Fund & $1970-2001$ & 3.90 \\
Vanguard Morgan Growth/Inv & $1968-2001$ & 4.40
\end{tabular}

Table 3: Individual mutual funds' posterior mean of alpha, from the newest to oldest, and who have at least a $95 \%$ chance of returning a market excess return greater than the average mutual fund fee of $1.5 \%$.

the funds possess the extraordinary ability to pick stocks such that they are expected to produce an alpha that is greater than $15 \%$ per year. The top performers are newer funds with less than seven years of experience. There are also a few highly skilled funds, like the Janus Fund, that have been in business since the 1970s. Hence, not all skilled funds suffer from the decreasing returns to scale hypothesized by Berk \& Green (2004).

\section{Conclusion}

With our Bayesian nonparametric learning approach, we find the posterior population distribution for the actively manage mutual fund industry to be fat-tailed, multi-modal, and skewed towards higher levels of skill. The population's primary mode is $1.8 \%$ per year, followed by a secondary mode at $-0.65 \%$, and lastly, a minor mode of $6 \%$. As a result of the location for the minor mode and the skewness of the population distribution towards 
higher skilled funds, a fund for which we have no information about has a greater chance of being skilled than previously thought. Under a parametric, normal, hierarchical, prior for alpha, the probability of finding an extraordinarily skilled fund is pushed back towards the primary mode; i.e., back toward a typical fund's alpha.

In comparison to when we treated skill idiosyncratically, our nonparametric approach finds fewer skilled and unskilled funds. Out of 5,136 funds, we find 22 (50) funds have a $95 \%$ chance of its alpha (not) exceeding the average fee charged by a fund of $1.5 \%$. Each of these exceptional funds has a unique posterior distribution relative to the population distribution where their likelihood function drives a wedge between its posterior for alpha and the posterior population distribution.

We found these highly skilled funds to be truly skilled and not just lucky. The posterior mean alphas were similar under both our nonparametric prior and the idiosyncratic priors, whereas, many of the funds that had larger posterior means under the idiosyncratic prior lacked the empirical performance to drive a wedge between their posterior and the nonparametric population distribution.

Learning the cross-sectional distribution of mutual fund skill with our Bayesian nonparametric approach can be applied to other related questions. For instance, any finance or economic problem where one is inferring a parameter and estimating the cross-sectional distribution of the parametric can be analyzed nonparametrically. For example, one can estimate how the multiple risk-factors' coefficients of an asset pricing model are distributed across different assets. We are currently investigating this and other similar types of research ideas with our Bayesian nonparametric learning approach. 


\section{References}

Avramov, D. \& Wermers, R. (2006), 'Investing in mutual funds when returns are predictable', Journal of Financial Economics 81, 339-377.

Baks, K. P., Metrick, A. \& Wachter, J. (2001), 'Should investors avoid all actively managed mutual funds? A study in Bayesian performance evaluation', Journal of Finance 56, $45-85$.

Barras, L., Gagliardini, P. \& Scaillet, O. (2018), The cross-section distribution of fund skill measures, Technical report, Université De Genéve.

Barras, L., Scaillet, O. \& Wermers, R. (2010), 'False discoveries in mutual fund performance: Measuring luck in estimated alphas', The Journal of Finance 65(1), 179-216.

Bassetti, F., Casarin, R. \& Leisen, F. (2014), 'Beta-product dependent Pitman-Yor processes for Bayesian inference', Journal of Econometrics 180(1), 49 - 72.

Berk, J. B. \& Green, R. C. (2004), 'Mutual fund flows and performance in rational markets', Journal of Political Economy 112, 1269-1295.

Bernardo, J. M. \& Smith, A. F. M. (2000), Bayesian Theory, Wiley.

Blackwell, D. \& MacQueen, J. (1973), 'Ferguson distributions via polya urn schemes', The Annals of Statistics 1, 353-355.

Burr, D. \& Doss, H. (2005), 'A Bayesian semiparametric model for random-effects metaanalysis', Journal of the American Statistical Association 100(469), 242-251.

Carhart, M. M. (1997), 'On persistence in mutual fund performance', The Journal of Finance $\mathbf{5 2}(1)$, pp. 57-82.

Carvalho, C. M., Lopes, H. F., Polson, N. G. \& Taddy, M. A. (2010), 'Particle learning for general mixtures', Bayesian Analysis 5(4), 709-740.

Chen, H.-L. \& Pennacchi, G. G. (2009), 'Does prior performance affect a mutual funds choice of risk? Theory and further empirical evidence', Journal of Financial and Quantitative Analysis 44, 745-775.

Chen, Y., Cliff, M. \& Zhao, H. (2017), 'Hedge funds: The good, the bad, and the lucky', Journal of Financial and Quantitative Analysis 52(3), 1081-1109. 
Chib, S. \& Hamilton, B. (2002), 'Semiparametric Bayes analysis of longitudinal data treatment models', Journal of Econometrics 110, 67-89.

Cohen, R. B., Coval, J. D. \& Pástor, L. (2005), 'Judging fund managers by the company they keep', The Journal of Finance $\mathbf{6 0}(3)$.

Dunson, D. B. (2010), Nonparametric Bayes application to biostatistics, in N. L. Hjort, C. Holmes, P. Mueller \& S. G. Walker, eds, 'Bayesian Nonparametrics', Cambridge University Press, pp. 223-268.

Elton, E. J. \& Gruber, M. J. (2013), Mutual Funds, Vol. 2 of Handbook of the Economics of Finance, Elsevier, pp. 1011 - 1061.

Escobar, M. D. \& West, M. (1995), 'Bayesian density estimation and inference using mixtures', Journal of the American Statistical Association 90(430), 577-588.

Fama, E. F. \& French, K. R. (1993), 'Common risk factors in the returns on stocks and bonds', Journal of Financial Economics 33(1), 3 - 56.

Fama, E. F. \& French, K. R. (2010), 'Luck versus skill in the cross-section of mutual fund returns', The Journal of Finance 65(5), 1915-1947.

Ferguson, T. (1973), 'A Bayesian analysis of some nonparametric problems', The Annals of Statistics 1(2), 209-230.

Ferson, W. \& Chen, Y. (2015), How many good and bad fund managers are there, really? Working Paper.

Gelman, A., Carlin, J. B., Stern, H. S., Dunson, D. B., Vehtari, A. \& Rubin, D. B. (2013), Bayesian Data Analysis, Chapman and Hall/CRC.

Gruber, M. J. (1996), 'Another puzzle: The growth in actively managed mutual funds', The Journal of Finance 51(3), pp. 783-810.

Harvey, C. R. \& Liu, Y. (2018), 'Detecting repeatable performance', The Review of Financial Studies 31(7), 2499-2552.

Hirano, K. (2002), 'Semiparametric Bayesian inference in autoregressive panel data models', Econometrica 70, 781-799.

Jensen, M. C. (1968), 'The performance of mutual funds in the period 1945-1964', The Journal of Finance 23(2), pp. 389-416. 
Jensen, M. J. (2004), 'Semiparametric Bayesian inference of long-memory stochastic volatility models', Journal of Time Series Analysis 25(6), 895-922.

Jensen, M. J. \& Maheu, J. M. (2010), 'Bayesian semiparametric stochastic volatility modeling', Journal of Econometrics 157(2), 306 - 316.

Jones, C. S. \& Shanken, J. (2005), 'Mutual fund performance with learning across funds', Journal of Financial Economics 78, 507-552.

Kleinman, K. P. \& Ibrahim, J. G. (1998), 'A semiparametric Bayesian approach to the random effects mode', Biometrics 54, 921-938.

Kosowski, R., Timmermann, A., Wermers, R. \& White, H. (2006), 'Can mutual fund "stars" really pick stocks? New evidence from a bootstrap analysis', The Journal of Finance 61(6), pp. 2551-2595.

Kothari, S. \& Warner, J. B. (2001), 'Evaluating mutual fund performance', The Journal of Finance 56(5), 1985-2010.

Muller, P. \& Rosner, G. L. (1997), 'A Bayesian population model with hierarchical mixture priors applied to blood count data', Journal of the American Statistical Association 92(440), 1279-1292.

Murphy, K. P. (2012), Machine Learning: A Probabilistic Perspective, MIT Press, Cambridge, Mass.

Neal, R. (2000), 'Markov chain sampling methods for Dirichlet process mixture models', Journal of Computational and Graphical Statistics 9, 249-265.

Ohlssen, D. I., Sharples, L. D. \& Spiegelhalter, D. J. (2007), 'Flexible random-effects models using Bayesian semi-parametric models: applications to institutional comparisons', Statistics in Medicine 26(9), 2088-2112.

Pástor, L. \& Stambaugh, R. F. (2002a), 'Investing in equity mutual funds', Journal of Financial Economics 63(3), 351 - 380.

Pástor, L. \& Stambaugh, R. F. (2002b), 'Mutual fund performance and seemingly unrelated assets', Journal of Financial Economics 63(3), 315 - 349.

Singh, S. \& Maddala, G. (1976), 'A function for size distribution of incomes', Econometrica 44, 963-970. 
Verbeke, G. \& Lesaffre, E. (1996), 'A linear mixed-effects model with heterogeneity in the random-effects population', Journal of the American Statistical Association 91(433), 217-221.

Wermers, R. (2011), 'Performance measrement of mutua funds, hedge funds, and institutional accounts', Annual Review of Finanacial Economics 3, 537-574.

West, M., Muller, P. \& Escobar, M. (1994), Hierarchical priors and mixture models with applications in regression and density estimation, in P. R. Freeman \& A. F. Smith, eds, 'Aspects of Uncertainty', John Wiley. 